Original research article

\title{
Comparative repeat profiling of two closely related conifers (Larix decidua and Larix kaempferi) reveals high genome similarity with only few fast-evolving satellite DNAs
}

\author{
Tony Heitkam**1, Luise Schulte ${ }^{1,2,3}$, Beatrice Weber ${ }^{1}$, Susan Liedtke ${ }^{1}$, Sarah \\ Breitenbach $^{1}$, Anja Kögler ${ }^{1}$, Kristin Morgenstern ${ }^{4}$, Marie Brückner ${ }^{5}$, Ute \\ Tröber $^{5}$, Heino Wolf ${ }^{5}$, Doris Krabel ${ }^{4}$, and Thomas Schmidt ${ }^{\dagger 1}$ \\ ${ }^{1}$ Institute of Botany, Technische Universität Dresden, 01069 Dresden, Germany \\ 2 Alfred Wegener Institute Potsdam, Telegrafenberg A43, 14473 Potsdam, Germany \\ (present affiliation) \\ ${ }^{3}$ Institute of Biochemistry and Biology, University of Potsdam, 14476 Potsdam, \\ Germany (present affiliation) \\ ${ }^{4}$ Institute of Forest Botany, Technische Universität Dresden, 01737 Tharandt, Germany \\ ${ }^{5}$ Staatsbetrieb Sachsenforst, 01796 Pirna OT Graupa, Germany
}

*Manuscript corresponding author:

Tony Heitkam

Institute of Botany

Technische Universität Dresden

01069 Dresden

Germany

Phone: (+49) 035146339593

E-Mail: tony.heitkam@tu-dresden.de

† Deceased 1 August 2019

Running title: Repetitive DNA in larches 


\section{ABSTRACT}

2 In eukaryotic genomes, cycles of repeat expansion and removal lead to large-scale 3 genomic changes and propel organisms forward in evolution. However, in conifers, 4 active repeat removal is thought to be limited, leading to expansions of their genomes, 5 mostly exceeding 10 gigabasepairs. As a result, conifer genomes are largely littered with 6 fragmented and decayed repeats. Here, we aim to investigate how the repeat landscapes 7 of two related conifers have diverged, given the conifers' accumulative genome 8 evolution mode. For this, we applied low coverage sequencing and read clustering to the 9 genomes of European and Japanese larch, Larix decidua (Lamb.) Carrière and Larix 10 kaempferi (Mill.), that arose from a common ancestor, but are now geographically 11 isolated. We found that both Larix species harbored largely similar repeat landscapes, 12 especially regarding the transposable element content. To pin down possible genomic 13 changes, we focused on the repeat class with the fastest sequence turnover: satellite 14 DNAs (satDNAs). Using comparative bioinformatics, Southern, and fluorescent in situ 15 hybridization, we reveal the satDNAs' organizational patterns, their abundances, and 16 chromosomal locations. Four out of the five identified satDNAs are widespread in the 17 Larix genus, with two even present in the more distantly related Pseudotsuga and Abies 18 genera. Unexpectedly, the EulaSat3 family was restricted to L. decidua and absent from 19 L. kaempferi, indicating its evolutionarily young age. Taken together, our results 20 exemplify how the accumulative genome evolution of conifers may limit the overall 21 divergence of repeats after speciation, producing only few repeat-induced genomic 22 novelties.

24 Keywords: Larix decidua, Larix kaempferi, satellite DNA, tandem repeats, 25 retrotransposon, repetitive DNA, fluorescent in situ hybridization (FISH) 


\section{INTRODUCTION}

27 Ranging in size between 0.002 and nearly $150 \mathrm{~Gb}$, eukaryotic genomes vary by several orders of magnitude (Hidalgo et al., 2017). Among those, conifer genomes are especially large with sizes up to $37 \mathrm{~Gb}$ (Ahuja and Neale, 2005). As new reference genome sequences are generated - among them conifers such as spruces, pines, and recently firs and larches - new insights into the composition of conifer genomes are brought forward (Nystedt et al., 2013; Wegrzyn et al., 2014; Stevens et al., 2016; Kuzmin et al., 2019; Mosca et al., 2019). One of the main takeaways is that the steady accumulation of repeats is the main driver for conifer genome expansion, presumably due to limited elimination of transposable elements (TEs; Nystedt et al., 2013; Prunier et al., 2016).

As the large conifer genomes have accumulated repeats over long periods of time with only slow removal and turnover of repetitive sequences, we wondered whether speciesspecific repeat profiles were able to evolve in closely related conifers. Regarding repetitive sequence classes, it is already hypothesized that TE families likely persist in conifers over long evolutionary timeframes (Zuccolo et al., 2015). In contrast, satellite DNAs (satDNAs) have much faster sequence turnovers than TEs. They form one of the major repeat groups, constituting up to $36 \%$ of some plant genomes (Ambrozová et al., 2011; Garrido-Ramos, 2017). SatDNAs are composed of short monomers with individual lengths often between 160-180 bp and 320-360 bp (Hemleben et al., 2007; Melters et al., 2013), and are arranged in long tandemly repeated arrays. As they confer important functions with roles in cell division, chromatid separation, and chromosome stability (Jagannathan et al., 2018), they often occupy specific chromosomal regions, such as the centromeres and the (sub-) telomeres (Schmidt and Heslop-Harrison, 1998; Melters et al., 2013; Oliveira and Torres, 2018). Due to their fast evolution and defined chromosomal localization, satDNAs may represent valuable targets to trace repeat evolution and divergence over long, evolutionary timeframes in conifers.

As models, we investigate two related conifers within the genus Larix, the deciduous

54 European and Japanese larches, i.e. Larix decidua (Lamb.) Carrière and Larix kaempferi 55 (Mill.). Their genome sizes range between $11 \mathrm{~Gb}$ (L. kaempferi) and $13 \mathrm{~Gb}$ ( $L$. 56 japonica) (Zonneveld, 2012; Bennett and Leitch, 2019), with their huge genomes likely 57 being the result of many divergent and ancient repeats (Nystedt et al., 2013; Pellicer et al., 2018). Larches frequently hybridize, leading to an unclear genetic basis with 
debated phylogenetic positions of individual species (Wei and Wang, 2003; Lu et al., 2014). From a breeding perspective, the interspecific hybrid Larix $\times$ eurolepis (with parental contributions of L. decidua and L. kaempferi) offers interesting possibilities for larch cultivation outside the natural range, especially in Europe (Pâques et al., 2013), however determining the parental contributions to the traits of larch hybrids remains difficult.

Consistent with other Pinaceae species, all larches have $2 n=2 x=24$ chromosomes with conserved sizes, divided into six meta- and six submetacentric chromosome pairs (Hizume et al., 1993; Prunier et al., 2016). In larches, the 18S-5.8S-25S and 5S rDNAs are physically separated (Garcia and Kovař́k, 2013), and fluorescent in situ hybridization (FISH) with respective rDNA probes clearly mark three and one chromosome pair for L. decidua (Lubaretz et al., 1996) and two and one pair for $L$. kaempferi (Liu et al., 2006; Zhang et al., 2010), respectively. Similarly, a single satDNA family is known ("LPD”), marking a heterochromatic chromosomal band on 22 chromosomes in L. kaempferi (Hizume et al., 2002). However, how the accumulative genome evolution mode of conifers affects the landscapes of larch repeats after speciation is not understood by far.

To test this, we sequenced L. decidua and L. kaempferi in low coverage to quantify, classify, and compare the respective repeat fractions. As satDNAs are typically marked by high sequence turnovers, we expect the highest differences for this repeat class. Using comparative bioinformatics, Southern, and fluorescent in situ hybridization, we deeply profiled five selected satDNAs, focusing on their abundance, their higher order arrangements, and chromosomal location. Assessment of their genomic distribution over a wider range of gymnosperms may give insight into the evolutionary age of satDNAs, may allow pinpointing how conifer repeat landscapes have diverged after speciation, and may be used to gather information regarding the parentage of larch hybrids.

\section{MATERIALS AND METHODS}

\section{Plant material and DNA isolation}

Needles and seeds of eleven gymnosperm accessions have been obtained from the Forestbotanical Garden Tharandt (Technische Universität Dresden) and the Staatsbetrieb Sachsenforst (Table 1). DNA was isolated from $2 \mathrm{~g}$ of homogenized 
92 material from frozen needles using the DNeasy Plant Maxi Kit (Qiagen, Hilden,

93 Germany) according to manufacturer's instructions. To allow for a more efficient

94 elution of conifer DNA, the incubation time during the elution step was increased to 10

minutes. Purified DNA was eluted into water instead of the provided AE buffer.

For cytogenetics, we have used primary root tips from seeds of L. decidua (obtained as selected material for propagation from Staatsdarre Flöha, Partie number 1846, ELA/83704) and L. kaempferi seeds (obtained from Niedersächsische Landesforsten, provenance number 83901), as well as roottips from L. $\times$ eurolepis plantlets (clone

100 56.012.15) obtained from somatic embryogenic cultures from Madlen Walter and Kurt Zoglauer from the Humboldt Universität zu Berlin.

\section{Sequencing, read clustering, repeat classification, and characterization}

104 For sequencing, we used L. decidua and L. kaempferi as reference, with accessions as

105 indicated in Table 1. Whole genome sequence libraries with 350 to $500 \mathrm{bp}$ fragment 106 sizes have been generated by Macrogen Inc., followed by Illumina paired end 107 sequencing on Illumina HiSeq2000 and HiSeq2500 machines. The reads were trimmed 108 to the same length (101 bp) using trimmomatic (Bolger et al., 2014). We pre-treated and 109 interlaced the read sequences using the custom scripts accompanying the local 110 RepeatExplorer installation (paired_fastq_filtering. $R$ and fasta_interlacer, followed by

111 seqclust). The reads were quality-trimmed to include only sequences with a Phred score $112 \geq 10$ over $95 \%$ of the read length. Overlapping paired ends have been excluded. We

113 randomly selected five million paired reads from each library and subjected those to 114 comparative clustering with the RepeatExplorer software (Novák et al., 2010; Novák et 115 al., 2013) and TAREAN (Novák et al., 2017). The resulting clusters were classified by 116 similarity searches against the Conserved Domain Database for the functional 117 annotation of proteins (Marchler-Bauer et al., 2011), RepBase Update (Jurka et al., 118 2005), the REXdb database (Neumann et al., 2019), and a custom library containing 119 ribosomal, telomeric and plastid sequences. Clusters connected by paired reads and 120 sharing a common annotation have been manually combined to superclusters. Graphic 121 representations as bar and pie charts have been produced with $R$ using the ggplot 2 122 library (Wickham, 2016).

123 Clusters with a satellite-typical star-like and circular graphical representation (Novák et 124 al., 2010) have been selected for further analysis. Putative monomers were manually 
125 detected on the RepeatExplorer-derived contigs as well as with the software Tandem

126 Repeats Finder (Benson, 1999). Using the putative circular monomers as a template, we

127 iteratively aligned the paired reads against these template sequences to derive more

128 robust consensus sequences (Data S1). Repeat sequences have been compared and

129 characterized using multiple sequence alignments and dotplots of monomers with the

130 packages MAFFT (Katoh and Standley, 2013) and FlexiDot (Seibt et al., 2018). General

131 sequence investigation was performed with the multi-purpose software Geneious6.1.8

132 (Kearse et al., 2012).

\section{Repeat quantification by comparative read mapping}

135 To determine the relative abundance of the selected Larix repeat families in other

136 gymnosperms, we complemented our own data (L. decidua and L. kaempferi) with

137 publicly available whole genome shotgun Illumina reads from twelve gymnosperms.

138 From the Pinaceae, these data sets include other larches (Larix sibirica, Larix gmelinii),

139 pines (Pinus taeda, P. sylvestris, and P. lambertiana), spruces (Picea abies, P. glauca,

140 and P. sitchensis), fir (Abies sibirica), and Douglas fir (Pseudotsuga menziesii). We also

141 analyzed the genomes of distantly related gymnosperms, such as yew (Taxus baccata)

142 and common juniper (Juniperus communis). The publicly available reads were obtained

143 from NCBI under the accession numbers SRR8555411, SRR2027118, SRR1054646,

144 ERR268439, SRR2027090, ERR268355, SRR1259615, SRR3100750, ERR268418,

145 ERR268427, and ERR268423. We randomly extracted three million paired reads, and

146 iteratively mapped them against the circular satDNA consensus until it remained

147 unchanged. This alignment to the consensus was performed with the Geneious6.1.8

148 mapping tools (using medium sensitivity parameters, Kearse et al., 2012). We

149 graphically represented mapping counts as bubble chart with $R$ and ggplot2 (Wickham,

150 2016).

\section{Repeat detection in genome assemblies}

153 If we detected EulaSat1 to EulaSat5 presence in additional genomes, we downloaded

154 the corresponding genome assemblies, if available. These included assemblies of

155 Pseudotsuga menziesii (Psme v.1.0 from treegenesdb.org, Neale et al., 2017) and Abies

156 alba (Abal v.1.1 from treegenesdb.org, Mosca et al., 2019).Using a local BLAST search

157 (Altschul et al., 1990), we have retrieved the five scaffolds with the most hits for each 
158 of the satDNA consensuses. In order to assess the organization of the satDNA families,

159 we visualized each scaffold as a dotplot. For visualization purposes, we extracted

160 representative $20 \mathrm{~kb}$ regions and generated FlexiDot dotplots (Seibt et al., 2018) with

161 the parameters $-k 18-S 4-c n-p 0-A 1.5-T 40-E 16$.

\section{PCR and cloning}

164 From the monomeric consensus sequences, outward facing primers have been designed

165 from the L. decidua reference (Table 2). For the amplification of satDNA probes for 166 Southern hybridization and FISH, PCR was carried out with the specific primer pairs.

167 PCR reactions with $50 \mathrm{ng}$ plasmid template were performed in $50 \mu \mathrm{l}$ volume containing 168 10x DreamTaq buffer and 2.5 units of DreamTaq polymerase (Promega). Standard PCR 169 conditions were $94{ }^{\circ} \mathrm{C}$ for $5 \mathrm{~min}$, followed by 35 cycles of $94{ }^{\circ} \mathrm{C}$ for $1 \mathrm{~min}$, primer170 specific annealing temperature for $30 \mathrm{sec}, 72{ }^{\circ} \mathrm{C}$ for $1 \mathrm{~min}$ and a final incubation time at $17172{ }^{\circ} \mathrm{C}$ for $5 \mathrm{~min}$. The resulting amplicons have been cloned into the pGEM-T vector 172 (Promega), followed by Sanger sequencing. The clones containing inserts most similar 173 to the satDNA consensus have been chosen for hybridization experiments.

\section{Southern blot hybridization}

176 For Southern blots, genomic DNA was restricted with enzymes specific for each tandem 177 repeat targeted, separated on $2 \%$ agarose gels and transferred onto Hybond-N+ nylon 178 membranes (GE Healthcare) by alkaline transfer. Hybridizations were performed 179 according to standard protocols using probes labelled with ${ }^{32} \mathrm{P}$ by random priming 180 (Sambrook et al., 1989). Filters were hybridized at $60{ }^{\circ} \mathrm{C}$ and washed at $60{ }^{\circ} \mathrm{C}$ for 10 $181 \min$ in $2 x \mathrm{SSC} / 0.1 \%$ SDS. Signals were detected by autoradiography.

\section{Probe labelling, chromosome preparation, and fluorescent in situ hybridization}

184 Sequenced satDNA clones have been used as template for PCR-based labelling with 185 biotin-16-dUTP.The probe pZR18S containing a $5066 \mathrm{bp}$ fragment of the sugar beet 186 18S-5.8S-25S rRNA gene (HE578879, Paesold et al., 2012) was labeled with DY-415 187 or DY-647-dUTP (Dyomics) by nick translation. The probe pXV1 (Schmidt et al., 188 1994) for the 5S rRNA gene was labeled with digoxygenin-11-dUTP by nick 189 translation. 
190 We prepared mitotic chromosomes from the meristems of young primary roots,

191 harvested shortly after germination. Prior to fixation in ethanol:chloroform:glacial acetic 192 acid (2:1:1), root tips were incubated either for $16 \mathrm{~h}$ in $2 \mathrm{mM}$ 8-hydroxyquinoline or for

1931 hour in nitrous oxide at 10 bar. Fixed plant material was digested for 0.5 to 1.5 hours 194 at $37^{\circ} \mathrm{C}$ in an enzyme mixture consisting of $2 \%(\mathrm{w} / \mathrm{v})$ cellulase from Aspergillus niger 195 (Sigma C1184), $4 \%$ (w/v) cellulase Onozuka R10 (Sigma 16419),0.5\% (w/v) 196 pectolyase from Aspergillus japonicus (Sigma) P-3026,1 \% (w/v) cytohelicase from 197 Helix pomatia (Sigma) C-8274, 1\% hemicellulase from Aspergillus niger(Sigma $198 \mathrm{H} 2125$ ), and $20 \%$ (v/v) pectinase from Aspergillus niger (Sigma P4716) in citrate 199 buffer ( $4 \mathrm{mM}$ citric acid and $6 \mathrm{mM}$ sodium citrate). The root tips have been washed and 200 transferred to a slide, before maceration with a needle in $45 \%$ glacial acetic acid. 201 Before the slide dried, the chromosomes have been fixed with methanol:glacial acetic 202 acid (3:1).

203 Before FISH, according to the amount of cytoplasm visible under light microscope, we 204 pre-treated the slides with $100 \mu \mathrm{g} / \mathrm{ml} \mathrm{RNase}$ in $2 \times \mathrm{SSC}$ for $30 \mathrm{~min}$, followed by $200 \mu \mathrm{l}$ 205 of $10 \mu \mathrm{g} / \mathrm{ml}$ pepsin in $10 \mathrm{mM} \mathrm{HCl}$ for 15 to $30 \mathrm{~min}$. Slides with abundant cytoplasm 206 were additionally treated for 10 min with proteinase K. FISH was performed according 207 to the protocol of Heslop-Harrison et al.(1991) with modifications as described 208 (Schmidt et al., 1994). Probes were hybridized with a stringency of $76 \%$ and 209 subsequently washed with a stringency of $79 \%$. The chromosome preparations were 210 counterstained with DAPI (4', 6'-diamidino-2-phenylindole) and mounted in antifade 211 solution (CitiFluor). Slides were examined with a fluorescence microscope (Zeiss 212 Axioplan 2 imaging) equipped with Zeiss Filter 09 (FITC), Zeiss Filter 15 (Cy3), Zeiss 213 Filter 26 (Cy5), AHF Filter F36-544 (aqua), and Zeiss Filter 02 (DAPI). Images were 214 acquired directly with the Applied Spectral Imaging v. 3.3 software coupled with the 215 high-resolution CCD camera ASI BV300-20A.

\section{Data access}

218 Whole genome shotgun Illumina sequences have been deposited at the European 219 Bioinformatics Institute (EBI) short read archive in the project PRJEB42507 220 (http://www.ebi.ac.uk/ena/data/view/PRJEB42507). Similarly, cloned sequences used 221 as Southern and FISH probes have been deposited at EBI with the accession numbers LR994496 to LR994500). 


\section{RESULTS}

\section{L. decidua and L. kaempferi show very similar repeat profiles}

226 To assess the genome composition of L. decidua and L. kaempferi, we obtained paired227 end Illumina whole genome shotgun sequences with fragment sizes of $500 \mathrm{bp}$. Five 228 million reads of each genome have been randomly chosen for comparative low coverage 229 clustering with RepeatExplorer. The software automatically chose 2,124,798 ( $L$. 230 decidua) and 2,125,214 (L. kaempferi) reads (corresponding to a genome coverage 231 between 1.6 and $1.9 \%$ ) and yielded estimates of the repetitive fraction of $69.0 \%$ for

232 L. decidua and $68.1 \%$ for L. kaempferi. We classified the read clusters according to 233 their repeat class and manually combined clusters connected by read pairs and similar 234 annotation to superclusters. This has led to similar repeat profiles for both genomes 235 (Figure 1): In particular, Ty3-gypsy retrotransposons (approx. $31 \%$ for both genomes) 236 made up the largest fraction, followed by Ty1-copia retrotransposons (both approx. $23724 \%$ ). Presentation of the first 214 read superclusters as a two-sided, comparative 238 barchart illustrates the high degree of genomic similarity between both genomes (Figure 239 1). With only few exceptions, the read clusters are equally abundant in L. decidua and 240 L. kaempferi, with gaps indicating absence of the repeat from one of the genomes. We 241 detected most variation in the amount of satellite DNA, with $3.2 \%$ for L. decidua and $242 \quad 2.0 \%$ for L. kaempferi (Figure 1, marked in red).

244 Larix tandem repeats vary in abundance and genome organization, with only 245 punctual differences between $L$. decidua and $L$. kaempferi

246 Six of the analyzed RepeatExplorer read clusters produced circular or star-shaped 247 layouts, typical for tandem repeats (Figure S1), representative of five satDNA families.

248 Using L. decidua as reference organism, we extracted sequences of the candidates, and 249 named them EulaSat1 to EulaSat5, short for European larch satellite. We refined the 250 monomer consensus sequences by iterative mapping of three million paired reads to 251 generate robust consensus sequences (Figure 2, Data S1), used for quantification and 252 primer generation. In order to verify the consensus sequence and to generate 253 hybridization probes, we amplified and cloned all five candidates from L. decidua. 254 SatDNA characteristics are summarized in Table 3, whereas a multi-sequence dotplot 255 shows the family and subfamily structure (Figure S2). 
256 To verify the head-to-tail organization of the five EulaSat repeat families, we

257 transferred restricted genomic L. decidua DNA onto Southern membranes. After 258 Southern hybridization of the EulaSat probes, we investigated the resulting 259 autoradiographs for presence of satDNA-typical ladder hybridization (Figure 3), 260 indicating repeat organization in long arrays. In detail, summarizing the computational 261 and molecular data, the five satDNAs are characterized as follows:

262 1) Comprising approx. $1 \%$ of both Larix genomes, EulaSat1 is the major satDNA family in larches. First described as LPD, it is an integral part of many Larix genomes (Hizume et al., 2002), forming conserved 173 bp monomer with a G/C content of $33 \%$. Six of the eleven enzymes tested produced a satellite-typical restriction ladder for EulaSat1, all supporting the monomer length of $173 \mathrm{bp}$ (Figure 3A). Clearest signals up to the tetra- and pentamers have been generated with DraI (lane 4) and AluI (lane 8), whereas the remaining enzymes such as HindIII (lane 2) released longer multimers up to the dodecamer. Although the EulaSat1 consensus contains a potential HpaII/MspI restriction site (indicated in Figure 2), we detected only high molecular weight signals, indicating a high degree of DNA methylation.

2) With a genomic representation of $0.46 \%$ and $0.22 \%$, EulaSat 2 is the second-most abundant satDNA family in L. decidua and L. kaempferi. EulaSat2 has a relatively high G/C content with $44 \%$ and consists of monomers with the satDNA-typical length of $148 \mathrm{bp}$. The EulaSat2 autoradiograph (Figure 3B) showed ladder-like patterns for five enzymes. FokI, MboI, and AluI (lanes 6-8) released the EulaSat2 monomer, supporting its length of $148 \mathrm{bp}$. DraI (lane 4) only produced weak monomeric signals, whereas $R s a I$ (lane 9) did not generate any monomeric bands,

3) The EulaSat3 family is divided into three subfamilies with similar features: The pointing to only weak restriction site conservation. Bands up to the undecamer were conserved 345 bp long monomers contain a generally low G/C content between $26 \%$ and $31 \%$. Out of all identified repeats, only the three EulaSat3 subfamilies are genome-specific, as their clusters contain only reads from L. decidua and none from L. kaempferi. Consensus sequences of all EulaSat3 subfamilies can be subdivided into a $178 \mathrm{bp}$ and a $167 \mathrm{bp}$ subunit with identities ranging between $45.5 \%$ and $48.3 \%$ (Figure S3), suggesting evolution by EulaSat3 reorganization into structures 
of higher order. EulaSat3 hybridization (Figure 3C) generated ladder-like patterns with different intensities in all lanes, with its monomeric length (345 bp) distinguishable in most cases. For two enzymes, DraI (lane 4) and AluI (lane 8), bands below the monomer size were visible. These additional bands can be explained by multiple restriction sites in the monomer (see Figure 2), giving rise to 163 and $182 \mathrm{bp}$ fragments (DraI) as well as 36, 176, 196 and 212 bp fragments (AluI). In addition, HpaII and MspI were able to cut EulaSat3, both producing identical, weak ladders (lanes 10-11), pointing to presence of at least some monomers without DNA methylation in the putative restriction site.

4) Similarly, for EulaSat4, we detected two subfamilies with different monomeric lengths. EulaSat4a has 203 bp monomers and is more abundant, supported by a mapping of 1,104 reads. In contrast, the less frequent EulaSat $4 b$ subfamily (supported by 696 reads) has a monomer length of 169 bp. We did not detect clear, canonical ladder patterns after hybridization of EulaSat4 (Figure 3D). However, signals as detected for BsmI (lane 3), MboI (lane 7), and RsaI (lane 9) can be explained by the recognition of both EulaSat 4 subfamilies by the Southern probe. As observed, a combination of 203 and 169 bp fragments leads to the complex ladder patterns with unequal step sizes.

5) Out of all identified satDNA families, EulaSat5 has the shortest monomer ( $87 \mathrm{bp}$ ) and the highest $\mathrm{G} / \mathrm{C}$ content $(50 \%)$. Although the monomer is short, this satDNA family makes up $0.35 \%$ and $0.18 \%$ of the L. decidua and the L. kaempferi genomes, respectively.EulaSat5 hybridization (Figure 3E) yielded ladder patterns for the three enzymes AIw26I, FokI and MboI (lanes 5-7). For MboI, a strong monomeric signal was detected, providing additional support for the monomer size of $87 \mathrm{bp}$ and for the high restriction site conservation within EulaSat5 arrays. Intense signals in the hexaand heptamer regions indicate arrays with higher order repeat structures. Hybridization of HpaII/MspI-restricted DNA did not reveal bands in the low

\section{Individual Larix decidua chromosomes show comparable satDNA localizations}

319 To determine the position of the satDNA families along Larix chromosomes, we prepared mitotic and interphase chromosomes from the L. decidua reference, and in situ hybridized them with biotin-labeled satDNA probes (Figure 4A-E): 
1) EulaSat1 hybridized to 18 from the 24 L. decidua chromosomes, co-localizing with the strongly DAPI-stained heterochromatic proximal bands (Figure 4A). EulaSat1's occurrence in the deep heterochromatin was confirmed by co-localization with DAPI-positive regions on interphase nuclei (Figure 4A).

2) For EulaSat2, we have observed presence on all chromosomes. The localization along the centromeric constriction of all chromosomes indicates EulaSat2's suitability to serve as a marker for the centromere (Figure 4B). As this position is depleted in DAPI staining, we assume that the EulaSat2 regions are only loosely packaged. At higher resolution, using interphase nuclei, we confirmed that EulaSat2 is largely excluded from the heterochromatin (Figure 4B).

3) The three remaining satDNA families, EulaSat3 to EulaSat5 are marked by a dispersed localization along all L. decidua chromosomes (Figure 4C-E). For EulaSat3, we identified a range of minor signals without exclusion of the centromeres. Nevertheless, we found an enrichment of EulaSat3 hybridization sites along the intercalary and distal chromosome regions. At interphases, we noted

4) This pattern is mirrored for EulaSat4. We found that most of the minor EulaSat4 signals were localized at the intercalary chromosome regions. The distal chromosome regions and the centromeric restrictions were not excluded, but only few chromosomes carried EulaSat4 signals at these regions. At interphases, most signals were localized in the DAPI-positive heterochromatin (Figure 4D).

5) EulaSat5 signals were scattered over the whole length of all chromosomes, with frequent enrichments at or near the (peri-)centromeric regions. The signals are often euchromatic, but without exclusion from the DAPI-positive heterochromatin (Figure

347 Taken together, whereas three of the satDNA probes (EulaSat3 to EulaSat5) are 348 dispersed along all chromosomes, EulaSat1 and EulaSat2 produce distinct signals, 349 limited to the heterochromatic band and the centromeric constriction, and produce clear chromosomal landmarks.

\section{Distribution, abundance, and genomic organization in related conifer genomes}

We used bioinformatics and experimental approaches to investigate the abundance and genomic organization of the EulaSat repeats in related species. Using a read mapping 
species (Figure 5), including four larches, three pines, three spruces, a fir, and a Douglas fir. As outgroups, we also analyzed DNA of more distantly related yew (Taxus baccata) and juniper (Juniperus communis) trees.

As read mapping may misrepresent the factual genome representation of repeats due to inherent G/C biases (Benjamini and Speed, 2012; Chen et al., 2013), we complemented our bioinformatics approach with an experimental verification. For this, we comparatively hybridized the satDNA probes onto restricted genomic DNA, and quantified the repeat abundance in eleven species (Figure 6). Our species sampling includes L. decidua, L. kaempferi, L. gmelinii, L. sibirica (lanes 1-4), and a single representative of additional gymnosperm genera: Pseudotsuga menziesii (lane 5), Pinus sylvestris (lane 6), Picea abies (lane 7), Abies sibirica (lane 8), Taxus baccata (lane 9), Juniperus communis (lane 10), and Ginkgo biloba (lane 11).

Both approaches show that EulaSat1, EulaSat2, EulaSat4, andEulaSat5 are present in all four Larix accessions analyzed, indicating their wide-spread occurrence throughout the genus (Figures 5, 6).

1) Especially EulaSat1 is highly abundant in all four Larix species, but without occurrence outside of the genus (Figure 5). Supporting this, EulaSat1 hybridization revealed clear ladder signals in the genus Larix, already after 25 min of exposition (Figure 6A). We observed similar patterns and signal strengths in all Larix species tested, indicating similar EulaSat1 monomer sizes with organization in long arrays across the genus. The remaining genomes did not produce any signal, pointing to

2) A similar high abundance in Larix sp. was detected for EulaSat2. EulaSat2 was also present in $P$. menziesii, but in lower quantity (Figure 5). After EulaSat2 hybridization, clear ladder-like pattern is visible for all larch species tested (Figure 6B), supporting the organization of similar-sized monomers in a tandem arrangement. In addition, for Pseudotsuga menziesii (lane 5), very weak signals corresponding to the dimer and trimer are distinguishable, becoming more prominent after longer exposure (not shown), without additional signals in any other lanes. Hybridization to L. gmelinii DNA (lane 3) does only produce faint monomeric and dimeric bands, and instead leads to many signals in the higher, multimeric region. As the L. gmelinii DNA was restricted completely, this indicates a less conserved AluI restriction site in the EulaSat2 satDNA. 
3) Computationally, the three EulaSat3 subfamilies have been analyzed individually, indicating considerable genomic impact only in $L$. decidua. We did not detect presence in L. kaempferi and L. gmelinii. For subfamilies EulaSat3a and EulaSat3c, only few L. sibirica hits mapped to the consensus, suggesting a reduced abundance in this genome. The other gymnosperm sequences tested did not contain any similarity to the EulaSat3 subfamilies (Figure 5). The patchy distribution across the Larix genus was also apparent experimentally (Figure 6C), with hybridization revealing exclusive signals in L. decidua and L. sibirica. In both species, the monomeric band constituted the strongest signal, suggesting the high conservation of the MboI restriction site within EulaSat3. In L. decidua the satDNA-typical ladder pattern was formed, whereas in L. sibirica the multimeric bands were absent. As the signals were still faint after 17 days of exposure, we conclude a relatively low abundance in both genomes.

4) Out of all satDNAs analyzed, the EulaSat4a and EulaSat4b subfamilies had the broadest distribution. Apart from their presence in the Larix genomes, they also populate $P$. menziesii and A. alba genomes. In all six EulaSat4-containing genomes EulaSat4a has been more abundant than EulaSat4b (Figure 5). Corroborating this, the corresponding autoradiograph showed signals in species of the Larix, Pseudotsuga, and Abies genera (Figure 6D, lanes 1-5, 8). The remaining Pinaceae species (Pinus sylvestris and Picea abies) did not carry any signals, with longer exposition time (seven days) not changing this result. Hybridization to the larches produced very similar patterns, pointing to similar genomic organization. In Abies sibirica (lane 8) the lowest band represents a double signal, presumably generated by conserved $\mathrm{MboI}$ restriction sites in the two EulaSat4 subrepeats (169 and 203 bp). However, hybridization to $P$. menziesii (lane 5) produced a stronger ladder with bands slightly shifted towards lower molecular weights, suggesting a small deletion within the EulaSat4 monomers in this species.

5) Read mappings indicate EulaSat5 restriction to Larix genomes, with highest abundance in L. decidua (Figure 5). However, the corresponding probe hybridized to the species of the Larix and the Pseudotsuga genera (Figure 6E, lanes 1-5). Signal patterns of the larch species tested resemble each other, with a relatively strong monomeric band, a fainter dimeric band, and a smear at a higher molecular weight. In P. menziesii (lane 5), the smear was overlaid by a very faint band at approximately 
$480 \mathrm{bp}$, indicating low abundance. Longer exposition (six days) of the autoradiograph did not reveal EulaSat5 in further species.

424 Experimental and computational approaches revealed that two satDNA families also occurred outside of the Larix genus, i.e. in Pseudotsuga (EulaSat2, EulaSat4) and Abies (EulaSat4). For both genera, genome assemblies were available (Neale et al., 2017; Mosca et al., 2019). We queried those with all satDNA consensuses, and deeply inspected the five scaffolds with the most satDNA hits in the genome assemblies of $P$. menziesii and A. alba:

430 In P. menziesii, we extracted long EulaSat2 arrays spanning scaffolds over a megabase, with and without higher order arrangements (Figure S4A). This indicates that the

432 EulaSat2 family, though less abundant (Figure 5, 6B) still plays a major role in this genome.

434 For EulaSat4, in P. menziesii, we detected some arrays over $20 \mathrm{~kb}$, often interrupted by 435 other repeats (Figure S4B). The arrays included variable monomers and different 436 homogenization with or without higher order. In A. alba, longer arrays have been 437 detected more frequently. Strikingly, we noticed less monomer variation, with stronger 438 homogenization and a higher abundance of EulaSat4a than EulaSat4b (Figure S4C).

439 Taken together, our three approaches (read mapping, analyses of genome assemblies, 440 and experimental quantification) corroborate the different abundances of the five 441 satDNA repeats in the gymnosperms. We confirmed presence of EulaSat1, EulaSat2, 442 EulaSat4, and EulaSat5 in all Larix genomes tested. EulaSat2 and EulaSat4 reside also 443 in more distantly related Pinaceae genomes. In contrast, experimental and bioinformatic 444 evidence support the young age of EulaSat3 that is restricted to Siberian and European 445 larches.

447 Only very few differences distinguish the chromosomes of $\boldsymbol{L}$. decidua from those of

\section{L. kaempferi}

449 We aimed to combine the information gained from in situ hybridization to L. decidua 450 chromosomes (Figure 4) as well as from the quantitative comparisons of conifer 451 genomes (Figures 5-6). We now asked, how L. decidua and L. kaempferi genomes differ 452 on a chromosomal scale and if this information can be used to determine the parentage 453 of individual chromosomes in hybrids. 
454 Therefore, we have comparatively hybridized the most promising tandem repeat 455 landmark probes onto metaphases of both larch species (Figure7A-D), including also 456 the 5S and 18S-5.8S-25S rDNA probes and the satDNAs EulaSat1 and EulaSat2.

457 To check how the rDNA tandem repeat loci compare, we investigated the localization of 458 the $5 \mathrm{~S}$ and $18 \mathrm{~S}-5.8 \mathrm{~S}-25 \mathrm{~S}$ rDNAs (Figure 7A-B). Both species harbor two 5S rDNA 459 sites (magenta), located distally at the chromosome arms. For the 18S-5.8S-25S rDNA, 460 we observed hybridization on three chromosome pairs for L. decidua, and two pairs for 461 L. kaempferi (green), all localized at the secondary constrictions of the chromosomes 462 (Figure 7A-B).

463 Regarding the EulaSat1 and EulaSat2 satDNA families, a comparative hybridization 464 onto L. decidua and L. kaempferi metaphases showed that the satDNA arrays bordered 465 for both species, but with limited co-localization (Figure 7C-D). Overall, the 466 comparison between the major satDNAs EulaSat1 and EulaSat2 yielded only very few 467 differences between both species.

468 We then shifted attention to the genome-specific, but dispersed EulaSat3 satDNA 469 family that may be used to discern the parentage of individual chromosomes in hybrids. 470 For this, we have prepared metaphases from Larix $\times$ eurolepis, a hybrid between $L$. 471 decidua and L. kaempferi (Figure 7E). Hybridization of the 5S (magenta) and 18S-5.8S472 25S rDNAs (green) have yielded two and five signals, respectively, with the uneven 473 18S-5.8S-25S rDNA site number being a testimony to the hybrid status of the 474 individual. The EulaSat3 hybridization yields chromosomes with dispersed EulaSat3 475 hybridization, indicating L. decidua heritage, as well as chromosomes without signals, 476 pointing to descendance from L. kaempferi. Nevertheless, due to the dispersed pattern, 477 theEulaSat3 satellite can only give clear parental information for few chromosomes and 478 should be complemented by additional markers, if any become available.

479 Summarizing, genomes and chromosomes of European and Japanese larches are very 480 similar, with only very few hallmark differences. These include the number in rDNA 481 sites and the genome-specific satDNA family EulaSat3.

DISCUSSION

484 Similar repeat profiles in European and Japanese larch genomes presumably result from repeat accumulation versus turnover

486 Large conifer genomes evolve only slowly and keep many of their genomic repeats 487 buried within the genomes. With only limited downsizing, we hypothesized that two 
488 closely related conifer genomes (such as those from European and Japanese larches) 489 may not accumulate many changes in their overall repeat landscapes. To test this, we 490 have investigated the repeat profiles of these related larch genomes, starting with a 491 broad repeat comparison and then focusing on the repeat class with the fastest sequence 492 turnover, the satDNAs.

493 We have applied short read sequencing followed by read clustering to efficiently gain 494 insights into both genomes' satDNA contents (as laid out by Weiss-Schneeweiss et al., 495 2015; Novák et al., 2017). This approach has been successfully used to characterize the 496 repeat landscapes of many non-model plant species as for example beans, various 497 grasses, camellias, crocuses, quinoa, and ferns (Cai et al., 2014; Heitkam et al., 2015; 498 Ávila Robledillo et al., 2018; Kirov et al., 2018; Liu et al., 2019; Schmidt et al., 2019; 499 Heitkam et al., 2020; Ribeiro et al., 2020), and also of non-model animals such as 500 locusts, grasshoppers, or fishes (Ruiz-Ruano et al., 2016; Ferretti et al., 2020; 501 Boštjančić et al., 2021). For larch genomes, we provided evidence that LTR 502 retrotransposons and derived fragments are their main components, well in line with 503 reports for the related pines and spruces (Kamm et al., 1996; Kossack and Kinlaw, 504 1999; Nystedt et al., 2013; Stevens et al., 2016; Perera et al., 2018).

505 As only highly repetitive sequences $\geq 90 \%$ are considered in the RepeatExplorer 506 cluster analysis, the size estimations of Larix repeat fractions (approximately $68 \%$ of 507 the analyzed genomes) are bound to be vast underrepresentations, excluding the more 508 fragmented repeats. Especially in large genomes, such as those of the conifers analyzed 509 here, fragmentation and slow repeat divergence lead to barely recognizable transposable 510 elements (TEs), often termed "dark matter" (Maumus and Quesneville, 2016). With 511 increasing genome sizes, these dark matter repeats accumulate, leading to the observed 512 and potentially misleading low repeat fraction estimates, as also recently highlighted by 513 Novák et al. (2020).

514 For L. decidua and L. kaempferi, we find overall strikingly similar repeat profiles, 515 especially regarding the TE content, without major differences between European and 516 Japanese larches. The similarities include both, repeat family and abundance. In line 517 with evidence from other conifers (Prunier et al., 2016), these results also suggest the 518 limited TE elimination, usually carried out by recombination, reshuffling, or removal as 519 aftermath to genomic rearrangements (Ma et al., 2004; Ren et al., 2018; Kögler et al., 520 2020; Maiwald et al., 2020). Along the same lines, we did not observe any 
521 transpositional bursts of amplification during the speciation of the larches. Thus, only

522 limited TE-induced genomic novelty has likely occurred in the larches' accumulative 523 genome landscapes.

525 Evolutionarily young and old satDNAs contribute to genomic novelty in giant larch 526 genomes

527 We asked whether the conifer's genomic background of transposable element 528 accumulation and fragmentation has impacted the evolution of satDNAs. These usually 529 evolve by continued rounds of mutation and fixation leading to relatively fast sequence 530 turnovers, even at structurally important chromosomal locations such as the centromeric 531 regions (Dawe, 2005; Plohl et al., 2012). Indeed, the satDNA abundances in the 532 analyzed larch genomes differed much more than the respective TE portions: We 533 estimated the total satDNA content of L. decidua to $3.2 \%$ and of L. kaempferi to $2.0 \%$, 534 corresponding to satDNA amounts of approximately 416 and $220 \mathrm{Mb}$, respectively. 535 Overall, the larch satDNA proportions are of the same order of magnitude as already 536 estimated from BACs and fosmids for the related pines (1\%; Wegrzyn et al., 2013; 537 Neale and Wheeler, 2019). However, compared to the large values the satDNA genome 538 fraction can occupy in angiosperms (up to 36 \%; Ambrozová et al., 2011), the relative 539 amount for larches is rather low, though also not uncommon for angiosperm genomes 540 (Garrido-Ramos, 2017).

541 To better understand the contribution of satDNA to the genomic differences in larches,

542 we investigated five satDNA families in detail. Here, we will discuss their evolutionary 543 trajectories ranging from the evolutionarily oldest families occurring in several conifer 544 genera (EulaSat2 and EulaSat4), over to those distributed only in the genus Larix 545 (EulaSat1 and EulaSat5), to the species-specific family EulaSat3:

546 The most widely distributed satDNA identified is EulaSat4, with occurrences in larches,

547 Douglas fir and Siberian fir. Interestingly, the comparative read mappings, Southern 548 hybridizations and analyses of available genome assemblies point to longer and more 549 homogenized EulaSat4 arrays in common and Douglas firs than those observed in 550 larches. We therefore think that EulaSat4 is an evolutionarily old repeat, probably 551 playing a larger role in the Abies and Pseudotsuga genomes. EulaSat4's patchy 552 distribution across the conifers is an example of a satDNA family's occurrence that is 553 incongruent with the species phylogeny. The satellite library hypothesis may explain 
554 this pattern, by assuming that a common set of satDNAs resides in genomes in low copy 555 numbers (Fry and Salser, 1977; Utsunomia et al., 2017; Palacios-Gimenez et al., 2020).

556 Different satDNA amplification would then lead to the observed patchy abundance 557 pattern of EulaSat4.These low-copy satDNAs may reside within transposable elements, 558 possibly using these for their conservation and amplification (McGurk and Barbash, 559 2018; Belyayev et al., 2020; Vondrak et al., 2020). EulaSat4's dispersed localization 560 along all L. decidua chromosomes as well as the complex RepeatExplorer cluster graphs may indicate such a retrotransposon association. As retrotransposons are strongly conserved across conifer species (Zuccolo et al., 2015; this report), it is likely that EulaSat4 has been retained within a transposable element, followed by patchy amplification in Larix, Pseudotsuga, and Abies species.

565 The EulaSat 2 family co-localizes with the primary constriction of the L. decidua and $L$.

566 kaempferi chromosomes, indicating a possible role in centromere formation. Although 567 some plants have centromeres that differ fundamentally from each other (Gong et al., 568 2012), that does not seem to be the case for larches. The centromeres of all 569 chromosomes harbor EulaSat2, indicating similar sequences and structures. The 148 bp 570 monomers of EulaSat2 are well in line with lengths observed for other centromeric satDNAs, such as that of rice (Zhang et al., 2013), and a bit shorter than the canonical $572 \sim 170$ bp monomers of the mammalian alpha satellite (Willard and Waye, 1987).

573 EulaSat2 is more abundant in L. decidua than in L. kaempferi, indicating recent array 574 size fluctuations. Nevertheless, EulaSat2 is evolutionarily older with presence in the 575 related Douglas fir, but absence from the more distantly related pine, spruce and fir species tested. In fact, centromeric satDNAs of related spruces have already been characterized and differ strongly from Eulasat2 in sequence and monomer length (305 bp; Sarri et al., 2008; Sarri et al., 2011).

In all larches analyzed, the most abundant satDNA is EulaSat1, also known as LPD

580 (Hizume et al., 2002). Its canonical monomer length of 173 bp is similar in all Larix 581 species analyzed, but was not detected outside the genus. It is generally assumed that 582 the most abundant satDNA localizes at the centromeres (Melters et al., 2013), however 583 some exceptions have been already reported, e.g. for camellias (Heitkam et al., 2015). 584 Instead of the expected centromeric locations, EulaSat1 constitutes the highly heterochromatic, DAPI-positive band present on most of its 24 chromosomes.

586 Regarding EulaSat1's evolution, our data indicates strong EulaSat1 amplification after 587 the split from Pseudotsuga. Interestingly, the different species set tested by Hizume et 
588 al. (2002) indicates also a patchy abundance in some Picea, Pinus, Abies, and Tsuga

589 species - claims that we cannot verify with our data. Nevertheless, we can convincingly

590 show that differences in abundance between $L$. decidua and L. kaempferi point to

591 EulaSat1 array expansions and reductions during the more recent evolutionary events.

592 In contrast to EulaSat1 and EulaSat2, only short arrays were detected for EulaSat5, the

593 second satDNA family restricted to the larches. In situ hybridization marked a scattered

594 localization along all chromosomes, typical for short satDNA arrays. As with EulaSat4,

595 an explanation for the short arrays may be an association with transposable elements.

596 We have observed partially mixed RepeatExplorer clusters that may point towards an

597 embedment within retrotransposons, also often observed for short satDNA arrays

598 (Meštrović et al., 2015; Satović et al., 2016; Belyayev et al., 2020; Sultana et al., 2020).

599 Similarly, concatenated TEs or part from TEs may have satDNA-like properties, but

600 tend to occur dispersedly along chromosomes (Maiwald et al., 2020; Vondrak et al.,

601 2020). Here, our data are not sufficient to conclusively resolve the large-scale

602 organization of EulaSat5 within larch genomes.

603 In contrast to all other families investigated, EulaSat3 has experienced a very recent 604 birth, indicating an evolutionarily young age. EulaSat3 is clearly absent from $L$. 605 kaempferi, but occurs in L. decidua with three subfamilies, all containing distinct 345

606 bp monomers. Their arrangement in higher order is detectable by close inspection of the

607 monomer consensuses and the autoradiograms after Southern hybridization, indicating

608 still ongoing homogenization. FISH and hybridization to HpaII/MspI-restricted genomic

609 DNA have indicated that at least some EulaSat3 monomers are embedded in

610 euchromatic regions. We speculate that these genomic regions are still actively

611 restructured and recombined; processes that potentially restrict the EulaSat3 array size.

612 Taken together, EulaSat3 is a relatively young satellite DNA and presumably marks the

613 evolutionarily young regions of L. decidua genomes.

614 To investigate whether EulaSat3 can be applied as a chromosome-specific marker of

615 L. decidua parentage in hybrid offspring, we have tested, if chromosome regions from

616 L. decidua can be identified in L. $\times$ eurolepis hybrids between L. kaempferi and

617 L. decidua. Although the in situ hybridization clearly marks some chromosome regions

618 as derived from L. decidua, this method is not as useful as hoped for the clear

619 differentiation of parentally derived regions along larch hybrid chromosomes. 
620 Nevertheless, EulaSat3's genome specificity within the Larix genus as well as the

621 differences in abundance for many of the remaining satDNAs indicates that even large,

622 highly repetitive genomes with slow sequence turnovers can yield new, evolutionarily

623 young repeats and generate sequence innovation to further genome evolution. If these

624 repeats also carry a phylogenetic signal and may be used for taxonomic means (e.g. as

625 suggested by Dodsworth et al., 2014) is still open.

627 Conclusion

628 As conifers largely accumulate transposable elements with only reduced active removal 629 processes, their genomes become huge, loaded with many fragmented, barely 630 recognizable repeat copies. As a result, we believe that closely related conifers harbor 631 very similar repeat landscapes. We have tested this hypothesis for two larch species and 632 detected highly similar TE profiles as well as very few differences in their tandem 633 repeat compositions. Nevertheless, despite the high overall repeat similarity, we 634 detected EulaSat3, a satDNA family present in European larches, but absent from their 635 Japanese counterparts. This illustrates that repeat-driven genome innovation still plays a 636 role, even in the huge, repetitive and fragmented conifer genomes.

637

\section{ACKNOWLEDGEMENTS}

639 We remember the late Thomas Schmidt, who has initiated the comparative analyses of 640 larch genomes and has made the ongoing cooperation between three of the partner 641 institutes listed here possible. We also keep in mind the late Ines Walter who has 642 established cytogenetics for larches in our lab. As a tribute, we have included her very 643 first, serendipitous FISH on larches (Figure 7A).

644 We gratefully acknowledge funding by the German Federal Ministry of Food and 645 Agriculture (Fachagentur Nachwachsende Rohstoffe e. V.; grants 22031714 and 646 22002216) to TS, DK, and HW. We thank the Forest Botanical Garden of Tharandt, the 647 Staatsbetrieb Sachsenforst, and Madlen Walther of the Humboldt Universität zu Berlin 648 for the contribution of plant material. We thank Ulrike Herzschuh (Alfred Wegener 649 Institute Potsdam) for providing pre-publication access to sequencing data of Larix 650 gmelinii, and we acknowledge Prof. Stefan Wanke for supporting our research initiative. 
651 Furthermore, we acknowledge the TU Dresden Center for Information Services and

652 High Performance Computing $(\mathrm{ZIH})$ for computer time allocations.

653

SHORT LEGENDS FOR SUPPORTING INFORMATION

655 Figure S1: Comparative clustering of L. decidua and L. kaempferi reads yields

656 satDNA-typical cluster graphs, representative for the EulaSat1 to EulaSat5 repeats.

657 Figure S2: All against all dotplots indicating similarity between the satDNA consensus

658 sequences.

659 Figure S3: Higher-order arrangement of EulaSat3 monomers.

660 Figure S4: Dotplots of satDNA-containing scaffolds of P. menziesii and A. alba.

661 Data S1: Consensus sequences of the EulaSat monomers in fasta format.

662

663 AUTHOR CONTRIBUTIONS

$664 \mathrm{TH}$ and TS coordinated the project and interpreted the results. TH, LS, and BW wrote 665 the manuscript, and all authors contributed, proof-read, and edited. TH and AK 666 coordinated the genome sequencing process. TH performed the bioinformatics analysis 667 and contributed to probe preparation. LS and BW performed Southern experiments, LS, 668 SL, and SB prepared metaphase spreads and performed FISH. KM, MB, UT, HW and 669 DK selected and provided plant material, and guided the project intellectually.

670

\section{DISCLOSURE DECLARATION}

672 The authors declare no conflict of interests.

673

674 


\section{TABLES}

676 Table 1: Plant material

\begin{tabular}{cllll}
\hline$\#$ & Species & Origin $^{\mathrm{a}}$ & Accession $^{\circ}$ & Plant family \\
\hline 1 & Larix decidua Mill. & $\mathrm{T}$ & $50^{\circ} 58^{\prime} 58.0^{\prime \prime} \mathrm{N} 13^{\circ} 23^{\prime} 44.4^{\prime \prime E}$ & Pinaceae \\
2 & Larix kaempferi (Lamb.) Carrière & $\mathrm{G}$ & $\# 1041$ & \\
3 & Larix gmelinii (Rupr.) Kuzen. & $\mathrm{G}$ & $\# 868$ & \\
4 & Larix sibirica Ledeb. & $\mathrm{G}$ & $\# 1340(5 / 18)$ & \\
5 & Pseudotsuga menziesii var. viridis & $\mathrm{T}$ & $\# 1014$ Indiv. 2 &
\end{tabular}

$677 \frac{\mathrm{T}}{11 \quad \text { Ginkgo biloba }} \mathrm{N} 50^{\circ} 58^{\prime} 53.5^{\prime \prime} ; \mathrm{E} 13^{\circ} 34^{\prime} 27.2^{\prime \prime} \quad$ Ginkgoaceae

678

679

680 Table 2: Primer pairs for the generation of satDNA clones

\begin{tabular}{lllll}
\hline Primer & Sequence & G/C [\%] & Length [bp] & Tm [ $\left.{ }^{\circ} \mathbf{C}\right]$ \\
\hline EulaSat1_F & GTATGCACATTCTACGTCATAACG & 41.7 & 24 & 59.3 \\
EulaSat1_R & GAATGCGCAAACTATAGAAAGTCG & 41.7 & 24 & 59.3 \\
EulaSat2_F & TCAAAGTTGAAATCGACCGTGC & 43.5 & 23 & 58.9 \\
EulaSat2_R & ATGTCACATTGGTAGACGAGCG & 50.0 & 22 & 60.3 \\
EulaSat3_F & GAATTTTTAGTGTGATTGTTCAGTAG & 29.6 & 27 & 57.4 \\
EulaSat3_R & GGTCAGAAATGTTAGCATAGTCG & 43.5 & 23 & 58.9 \\
EulaSat4_F & GGCACAAGCTCAAGGTATAAGC & 50.0 & 22 & 60.3 \\
EulaSat4_R & ATGGCACAAGATCAAGGAAAGC & 45.5 & 22 & 58.4 \\
EulaSat5_F & TTCATTCTCGGAGACCTCACG & 52.4 & 21 & 59.8 \\
EulaSat5_R & GTCCTTAGTGGACAGTTGAGG & 52.4 & 21 & 59.8 \\
\hline
\end{tabular}

681

6 Pinus sylvestris

7 Picea abies (L.) H. Karst. $\quad$ T $\quad\left[{ }^{*} P f\right.$ 1935/35] 217/12

8 Abies sibirica Ledeb. $\quad$ [U/1] 403 a210 Indiv. left

9 Taxus baccata L. $\quad \mathrm{T} \quad$ [U/7] $401 \mathrm{c} 19$

10 Juniperus communis $L$. $\left.\quad T \quad{ }^{*} 2000 / 117\right] 837 / 1684 \quad$ Cupressaceae

682

683 Table 3: Characteristics of satDNA in L. decidua $(L d)$ and L. kaempferi $(L k)$ genomes.

\begin{tabular}{|c|c|c|c|c|c|c|c|c|c|c|}
\hline \multirow[t]{2}{*}{ Family } & \multicolumn{2}{|c|}{$\begin{array}{c}\text { Genome } \\
\text { proportion }[\%]^{\mathrm{a}}\end{array}$} & \multicolumn{2}{|c|}{$\begin{array}{l}\text { Monomer } \\
\text { length [bp] }\end{array}$} & \multicolumn{2}{|c|}{$\begin{array}{c}\text { G/C content } \\
{[\%]}\end{array}$} & \multicolumn{2}{|c|}{$\begin{array}{c}\text { Mean pairwise } \\
\text { identity [\%] }\end{array}$} & \multicolumn{2}{|c|}{ Read support } \\
\hline & $L d$ & $L k$ & $L d$ & $L k$ & $L d$ & $L k$ & Ld & $L k$ & $L d$ & $L k$ \\
\hline EulaSat1 & 1.28 & 0.81 & 173 & 173 & 33 & 33 & 91.8 & 90.3 & 74,373 & 46,173 \\
\hline EulaSat2 & 0.46 & 0.22 & 148 & 148 & 45 & 45 & 84.7 & 83.9 & 22,881 & 10,714 \\
\hline EulaSat3a & & & 345 & 345 & 28 & 28 & 98.0 & -- & 1,532 & 0 \\
\hline EulaSat3b & 0.05 & 0.00 & 345 & 345 & 31 & 31 & 94.2 & --- & 978 & 0 \\
\hline EulaSat3c & & & 345 & 345 & 26 & 26 & 95.2 & --- & 605 & 0 \\
\hline EulaSat4a & & & 203 & 203 & 43 & 43 & 86.6 & 85.8 & 1,104 & 696 \\
\hline EulaSat4b & 0.09 & 0.08 & 169 & 169 & 43 & 43 & 86.8 & 86.3 & 264 & 119 \\
\hline EulaSat5 & 0.35 & 0.18 & 87 & 87 & 50 & 50 & 87.1 & 84.8 & 4,510 & 1,398 \\
\hline
\end{tabular}

$684 \quad{ }^{a}$ RepeatExplorer-based estimate

685 b Number of reads mapping out of three million paired end reads 
686

687

688

689

690

691

692

693

694

695

696

697

698

699

700

701

702

703

704

705

706

707

708

709

710

711

712

713

714

715

716

717

718

719

720

721

722

723

724

725

726

727

728

729

730

731

732

733

734

735

736

\section{REFERENCES}

Ahuja, M.R., and Neale, D.B. (2005). Evolution of genome size in conifers. Silvae Genet. 54, 126-137.

Altschul, S.F., Gish, W., Miller, W., Myers, E.W., and Lipman, D.J. (1990). Basic local alignment search tool. J. Mol. Biol. 215, 403-410.

Ambrozová, K., Mandáková, T., Bures, P., Neumann, P., Leitch, I.J., Koblízková, A., Macas, J., and Lysak, M.A. (2011). Diverse retrotransposon families and an ATrich satellite DNA revealed in giant genomes of Fritillaria lilies. Ann. Bot. 107, 255-268.

Ávila Robledillo, L., Koblížková, A., Novák, P., Böttinger, K., Vrbová, I., Neumann, P., Schubert, I., and Macas, J. (2018). Satellite DNA in Vicia faba is characterized by remarkable diversity in its sequence composition, association with centromeres, and replication timing. Sci. Rep. 8, 5838.

Belyayev, A., Josefiová, J., Jandová, M., Mahelka, V., Krak, K., and Mandák, B. (2020). Transposons and satellite DNA: on the origin of the major satellite DNA family in the Chenopodium genome. Mob. DNA 11, 20.

Benjamini, Y., and Speed, T.P. (2012). Summarizing and correcting the GC content bias in high-throughput sequencing. Nucleic Acids Res. 40, e72-e72.

Bennett, M.D., and Leitch, I.J. (2019). Plant DNA C-values database (release 7.1, Apr 2019). http://www.kew.org/cvalues/.

Benson, G. (1999). Tandem repeats finder: A program to analyze DNA sequences. Nucleic Acids Res. 27, 573-580.

Bolger, A.M., Lohse, M., and Usadel, B. (2014). Trimmomatic: A flexible trimmer for Illumina sequence data. Bioinformatics 30, 2114-2120.

Boštjančić, L.L., Bonassin, L., Anušić, L., Lovrenčić, L., Besendorfer, V., Maguire, I., Grandjean, F., Austin, C.M., Greve, C., Hamadou, A.B., and Mlinarec, J. (2021). The Pontastacus leptodactylus (Astacidae) repeatome provides insight into genome evolution and reveals remarkable diversity of satellite DNA. Front. Genet. 11, 1820.

Cai, Z., Liu, H., He, Q., Pu, M., Chen, J., Lai, J., Li, X., and Jin, W. (2014). Differential genome evolution and speciation of Coix lacryma-jobi L. and Coix aquatica Roxb. hybrid guangxi revealed by repetitive sequence analysis and fine karyotyping. BMC Genomics 15, 1-16.

Chen, Y.C., Liu, T., Yu, C.H., Chiang, T.Y., and Hwang, C.C. (2013). Effects of GC bias in next-generation-sequencing data on de novo genome assembly. PLoS ONE 8, e62856.

Dawe, R.K. (2005). Centromere renewal and replacement in the plant kingdom. Proc. Natl. Acad. Sci. USA 102, 11573-11574.

Dodsworth, S., Chase, M.W., Kelly, L.J., Leitch, I.J., Macas, J., Novák, P., Piednoël, M., Weiss-Schneeweiss, H., and Leitch, A.R. (2014). Genomic repeat abundances contain phylogenetic signal. Syst. Biol.

Ferretti, A.B.S.M., Milani, D., Palacios-Gimenez, O.M., Ruiz-Ruano, F.J., and CabralDe-Mello, D.C. (2020). High dynamism for neo-sex chromosomes: satellite DNAs reveal complex evolution in a grasshopper. Heredity 125, 124-137.

Fry, K., and Salser, W. (1977). Nucleotide sequences of HS-alpha satellite DNA from kangaroo rat Dipodomys ordii and characterization of similar sequences in other rodents. Cell 12, 1069-1084.

Garcia, S., and Kovařík, A. (2013). Dancing together and separate again: gymnosperms exhibit frequent changes of fundamental $5 \mathrm{~S}$ and $35 \mathrm{~S}$ rRNA gene (rDNA) organisation. Heredity 111, 23-33.

Garrido-Ramos, A.M. (2017). Satellite DNA: An Evolving Topic. Genes 8, 1-41. 
Gong, Z., Wu, Y., Koblizkova, A., Torres, G.A., Wang, K., Iovene, M., Neumann, P., Zhang, W., Novak, P., Buell, C.R., Macas, J., and Jiang, J. (2012). Repeatless and repeat-based centromeres in potato: Implications for centromere evolution. Plant Cell 24, 3559-3574.

Heitkam, T., Petrasch, S., Zakrzewski, F., Kögler, A., Wenke, T., Wanke, S., and Schmidt, T. (2015). Next-generation sequencing reveals differentially amplified tandem repeats as a major genome component of Northern Europe's oldest Camellia japonica. Chromosome Res. 23, 791-806.

Heitkam, T., Weber, B., Walter, I., Liedtke, S., Ost, C., and Schmidt, T. (2020). Satellite DNA landscapes after allotetraploidisation of quinoa (Chenopodium quinoa) reveal unique A and B subgenomes. Plant J. 103, 32-52.

Hemleben, V., Kovarik, A., Torres-Ruiz, R.A., Volkov, R.A., and Beridze, T. (2007). Plant highly repeated satellite DNA: Molecular evolution, distribution and use for identification of hybrids. Syst. Biodivers. 5, 277-289.

Heslop-Harrison, J.S., Schwarzacher, T., Anamthawat-Jónsson, K., Leitch, A.R., Shi, M., and Leitch, I.J. (1991). In-situ hybridization with automated chromosome denaturation. Technique 3, 109-116.

Hidalgo, O., Pellicer, J., Christenhusz, M., Schneider, H., Leitch, A.R., and Leitch, I.J. (2017). Is There an Upper Limit to Genome Size? Trends Plant Sci. 22, 567573.

Hizume, M., Shibata, F., Matsumoto, A., Maruyama, Y., Hayashi, E., Kondo, T., Kondo, K., Zhang, S., and Hong, D. (2002). Tandem repeat DNA localizing on the proximal DAPI bands of chromosomes in Larix, Pinaceae. Genome 45, 777783.

Hizume, M., Tominaga, H.H., Kondo, K., Gu, Z., and Yue, Z. (1993). Fluorescent chromosome banding in six taxa of Eurasian Larix, Pinaceae. La Kromosomo II 69, 2342-2354.

Jagannathan, M., Cummings, R., and Yamashita, Y.M. (2018). A conserved function for pericentromeric satellite DNA. eLife 7, e34122.

Jurka, J., Kapitonov, V.V., Pavlicek, A., Klonowski, P., Kohany, O., and Walichiewicz, J. (2005). Repbase Update, a database of eukaryotic repetitive elements. Cytogenet. Genome Res. 110, 462-467.

Kamm, A., Doudrick, R.L., Heslop-Harrison, J.S., and Schmidt, T. (1996). The genomic and physical organization of Ty1-copia-like sequences as a component of large genomes in Pinus elliottii var. elliottii and other gymnosperms. Proc. Natl. Acad. Sci. USA 93, 2708-2713.

Katoh, K., and Standley, D.M. (2013). MAFFT Multiple sequence alignment software version 7: Improvements in performance and usability. Mol. Biol. Evol. 30, 772780.

Kearse, M., Moir, R., Wilson, A., Stones-Havas, S., Cheung, M., Sturrock, S., Buxton, S., Cooper, A., Markowitz, S., Duran, C., Thierer, T., Ashton, B., Mentjies, P., and Drummond, A. (2012). Geneious Basic: An integrated and extendable desktop software platform for the organization and analysis of sequence data. Bioinformatics 28, 1647-1649.

Kirov, I.V., Gilyok, M., Knyazev, A., and Fesenko, I. (2018). Pilot satellitome analysis of the model plant, Physcomitrella patens, revealed a transcribed and high-copy IGS related tandem repeat. Comp. Cytogenet. 12, 493-513.

Kögler, A., Seibt, K.M., Heitkam, T., Morgenstern, K., Reiche, B., Brückner, M., Wolf, H., Krabel, D., and Schmidt, T. (2020). Divergence of 3' ends as driver of Short Interspersed Nuclear Element (SINE) evolution in the Salicaceae. Plant J. 103, 443-458. 
Kossack, D.S., and Kinlaw, C.S. (1999). IFG, a gypsy-like retrotransposon in Pinus (Pinaceae), has an extensive history in pines. Plant Mol. Biol. 39, 417-426. Kuzmin, D.A., Feranchuk, S.I., Sharov, V.V., Cybin, A.N., Makolov, S.V., Putintseva, Y.A., Oreshkova, N.V., and Krutovsky, K.V. (2019). Stepwise large genome assembly approach: A case of Siberian larch (Larix sibirica Ledeb). BMC Bioinformatics 20, 37.

Liu, B., Zhang, S.G., Zhang, Y., Lan, T.Y., Qi, L.W., and Song, W.Q. (2006). Molecular cytogenetic analysis of four Larix species by bicolor fluorescence in situ hybridization and DAPI banding. Int. J. Plant Sci. 167, 367-372.

Liu, Q., Li, X., Zhou, X., Li, M., Zhang, F., Schwarzacher, T., and Heslop-Harrison, J.S. (2019). The repetitive DNA landscape in Avena (Poaceae): Chromosome and genome evolution defined by major repeat classes in whole-genome sequence reads. BMC Plant Biol. 19, 226.

Lu, Y., Ran, J.-H., Guo, D.-M., Yang, Z.-Y., and Wang, X.-Q. (2014). Phylogeny and divergence times of gymnosperms inferred from single-copy nuclear genes. PLOS ONE 9, e107679.

Lubaretz, O., Fuchs, J., Ahne, R., Meister, A., and Schubert, I. (1996). Karyotyping of three Pinaceae species via fluorescent in situ hybridization and computer-aided chromosome analysis. Theor. Appl. Genet. 92, 411-416.

Ma, J., Devos, K.M., and Bennetzen, J.L. (2004). Analyses of LTR-retrotransposon structures reveal recent and rapid genomic DNA loss in rice. Genome Res. 14, 860-869.

Maiwald, S., Weber, B., Seibt, K.M., Schmidt, T., and Heitkam, T. (2020). The Cassandra retrotransposon landscape in sugar beet (Beta vulgaris) and related Amaranthaceae: Recombination and re-shuffling lead to a high structural variability. Ann. Bot. 127, 91-109.

Marchler-Bauer, A., Lu, S., Anderson, J.B., Chitsaz, F., Derbyshire, M.K., DeweeseScott, C., Fong, J.H., Geer, L.Y., Geer, R.C., Gonzales, N.R., Gwadz, M., Hurwitz, D.I., Jackson, J.D., Ke, Z., Lanczycki, C.J., Lu, F., Marchler, G.H., Mullokandov, M., Omelchenko, M.V., Robertson, C.L., Song, J.S., Thanki, N., Yamashita, R.A., Zhang, D., Zhang, N., Zheng, C., and Bryant, S.H. (2011). CDD: A Conserved Domain Database for the functional annotation of proteins. Nucleic Acids Res. 39, D225-229.

Maumus, F., and Quesneville, H. (2016). Impact and insights from ancient repetitive elements in plant genomes. Curr. Opin. Plant Biol. 30, 41-46.

Mcgurk, M.P., and Barbash, D.A. (2018). Double insertion of transposable elements provides a substrate for the evolution of satellite DNA. Genome Res. 28, 714725.

Melters, D.P., Bradnam, K.R., Young, H.A., Telis, N., May, M.R., Ruby, J.G., Sebra, R., Peluso, P., Eid, J., Rank, D., Garcia, J.F., Derisi, J.L., Smith, T., Tobias, C., Ross-Ibarra, J., Korf, I., and Chan, S.W. (2013). Comparative analysis of tandem repeats from hundreds of species reveals unique insights into centromere evolution. Genome Biol. 14, R10.

Meštrović, N., Mravinac, B., Pavlek, M., Vojvoda-Zeljko, T., Šatović, E., and Plohl, M. (2015). Structural and functional liaisons between transposable elements and satellite DNAs. Chromosome Res. 23, 583-596.

Mosca, E., Cruz, F., Gómez-Garrido, J., Bianco, L., Rellstab, C., Brodbeck, S., Csilléry, K., Fady, B., Fladung, M., Fussi, B., Gömöry, D., González-Martínez, S.C., Grivet, D., Gut, M., Hansen, O.K., Heer, K., Kaya, Z., Krutovsky, K.V., Kersten, B., Liepelt, S., Opgenoorth, L., Sperisen, C., Ullrich, K.K., Vendramin, G.G., Westergren, M., Ziegenhagen, B., Alioto, T., Gugerli, F., Heinze, B., 
Höhn, M., Troggio, M., and Neale, D.B. (2019). A reference genome sequence for the European silver fir (Abies alba Mill.): A community-generated genomic resource. G3: Genes Genom. Genet. 9, 2039.

Neale, D.B., Mcguire, P.E., Wheeler, N.C., Stevens, K.A., Crepeau, M.W., Cardeno, C., Zimin, A.V., Puiu, D., Pertea, G.M., Sezen, U.U., Casola, C., Koralewski, T.E., Paul, R., Gonzalez-Ibeas, D., Zaman, S., Cronn, R., Yandell, M., Holt, C., Langley, C.H., Yorke, J.A., Salzberg, S.L., and Wegrzyn, J.L. (2017). The douglas-fir genome sequence reveals specialization of the photosynthetic apparatus in Pinaceae. G3: Genes Genom. Genet. 7, 3157-3167.

Neale, D.B., and Wheeler, N.C. (2019). "Noncoding and repetitive DNA," in The Conifers: Genomes, Variation and Evolution, eds. D.B. Neale \& N.C. Wheeler. (Cham: Springer International Publishing), 61-74.

Neumann, P., Novák, P., Hoštáková, N., and Macas, J. (2019). Systematic survey of plant LTR-retrotransposons elucidates phylogenetic relationships of their polyprotein domains and provides a reference for element classification. Mob. DNA 10, 1-17.

Novák, P., Guignard, M.S., Neumann, P., Kelly, L.J., Mlinarec, J., Koblížková, A., Dodsworth, S., Kovařík, A., Pellicer, J., Wang, W., Macas, J., Leitch, I.J., and Leitch, A.R. (2020). Repeat-sequence turnover shifts fundamentally in species with large genomes. Nature Plants 6, 1325-1329.

Novák, P., Neumann, P., and Macas, J. (2010). Graph-based clustering and characterization of repetitive sequences in next-generation sequencing data. BMC Bioinformatics 11, 378.

Novák, P., Neumann, P., Pech, J., Steinhaisl, J., and Macas, J. (2013). RepeatExplorer: A Galaxy-based web server for genome-wide characterization of eukaryotic repetitive elements from next-generation sequence reads. Bioinformatics 29, 792-793.

Novák, P., Robledillo, L.A., Koblızková, A., Vrbová, I., Neumann, P., and Macas, J. (2017). TAREAN: a computational tool for identification and characterization of satellite DNA from unassembled short reads. Nucleic Acids Res. 45, e111.

Nystedt, B., Street, N.R., Wetterbom, A., Zuccolo, A., Lin, Y.-C., Scofield, D.G., Vezzi, F., Delhomme, N., Giacomello, S., Alexeyenko, A., Vicedomini, R., Sahlin, K., Sherwood, E., Elfstrand, M., Gramzow, L., Holmberg, K., Hallman, J., Keech, O., Klasson, L., Koriabine, M., Kucukoglu, M., Kaller, M., Luthman, J., Lysholm, F., Niittyla, T., Olson, A., Rilakovic, N., Ritland, C., Rossello, J.A., Sena, J., Svensson, T., Talavera-Lopez, C., Theiszen, G., Tuominen, H., Vanneste, K., Wu, Z.-Q., Zhang, B., Zerbe, P., Arvestad, L., Bhalerao, R., Bohlmann, J., Bousquet, J., Garcia Gil, R., Hvidsten, T.R., De Jong, P., Mackay, J., Morgante, M., Ritland, K., Sundberg, B., Lee Thompson, S., Van De Peer, Y., Andersson, B., Nilsson, O., Ingvarsson, P.K., Lundeberg, J., and Jansson, S. (2013). The Norway spruce genome sequence and conifer genome evolution. Nature 497, 579-584.

Oliveira, L.C., and Torres, G.A. (2018). Plant centromeres: Genetics, epigenetics and evolution. Mol. Biol. Rep. 45, 1491-1497.

Paesold, S., Borchardt, D., Schmidt, T., and Dechyeva, D. (2012). A sugar beet (Beta vulgaris L.) reference FISH karyotype for chromosome and chromosome-arm identification, integration of genetic linkage groups and analysis of major repeat family distribution. Plant J. 72, 600-611.

Palacios-Gimenez, O.M., Milani, D., Song, H., Marti, D.A., Lopez-Leon, M.D., RuizRuano, F.J., Camacho, J.P.M., and Cabral-De-Mello, D.C. (2020). Eight million years of satellite DNA evolution in grasshoppers of the genus Schistocerca 
illuminate the ins and outs of the library hypothesis. Genome Biol. Evol. 12, 88102.

Pâques, L.E., Foffova, E., Heinze, B., Lelu-Walter, M.-A., Liesebach, M., and Philippe, G. (2013). "Larches (Larix sp.)," in Forest Tree Breeding in Europe, ed. L.E. Pâques. Springer Netherlands), 13-106.

Pellicer, J., Hidalgo, O., Dodsworth, S., and Leitch, I.J. (2018). Genome size diversity and its impact on the evolution of land plants. Genes 9, 88.

Perera, D., Magbanua, Z.V., Thummasuwan, S., Mukherjee, D., Arick, M., 2nd, Chouvarine, P., Nairn, C.J., Schmutz, J., Grimwood, J., Dean, J.F.D., and Peterson, D.G. (2018). Exploring the loblolly pine (Pinus taeda L.) genome by BAC sequencing and $c 0 t$ analysis. Gene 663, 165-177.

Plohl, M., Mestrovic, N., and Mravinac, B. (2012). Satellite DNA evolution. Genome Dyn. 7, 126-152.

Prunier, J., Verta, J.-P., and Mackay, J.J. (2016). Conifer genomics and adaptation: at the crossroads of genetic diversity and genome function. New Phytol. 209, 4462.

Ren, L., Huang, W., Cannon, E.K.S., Bertioli, D.J., and Cannon, S.B. (2018). A mechanism for genome size reduction following genomic rearrangements. Front. Genet. 9, 454-454.

Ribeiro, T., Vasconcelos, E., Dos Santos, K.G.B., Vaio, M., Brasileiro-Vidal, A.C., and Pedrosa-Harand, A. (2020). Diversity of repetitive sequences within compact genomes of Phaseolus L. beans and allied genera Cajanus L. and Vigna Savi. Chromosome Res. 28, 139-153.

Ruiz-Ruano, F.J., López-León, M.D., Cabrero, J., and Camacho, J.P.M. (2016). Highthroughput analysis of the satellitome illuminates satellite DNA evolution. Sci. Rep. 6, 28333.

Sambrook, J., Fritsch, E.F., and Maniatis, T. (1989). Molecular cloning: A laboratory manual. New York, USA: Cold Spring Harbor Laboratory Press.

Sarri, V., Ceccarelli, M., and Cionini, P.G. (2011). Quantitative evolution of transposable and satellite DNA sequences in Picea species. Genome 54, 431435.

Sarri, V., Minelli, S., Panara, F., Morgante, M., Jurman, I., Zuccolo, A., and Cionini, P.G. (2008). Characterization and chromosomal organization of satellite DNA sequences in Picea abies. Genome 51, 705-713.

Satović, E., Vojvoda Zeljko, T., Luchetti, A., Mantovani, B., and Plohl, M. (2016). Adjacent sequences disclose potential for intra-genomic dispersal of satellite DNA repeats and suggest a complex network with transposable elements. BMC Genomics 17, 997.

Schmidt, T., Heitkam, T., Liedtke, S., Schubert, V., and Menzel, G. (2019). Adding color to a century-old enigma: Multi-color chromosome identification unravels the autotriploid nature of saffron (Crocus sativus) as a hybrid of wild Crocus cartwrightianus cytotypes. New Phytol. 222, 1965-1980.

Schmidt, T., and Heslop-Harrison, J.S. (1998). Genomes, genes and junk: the largescale organization of plant chromosomes. Trends Plant Sci. 3, 195-199.

Schmidt, T., Schwarzacher, T., and Heslop-Harrison, J.S. (1994). Physical mapping of rRNA genes by fluorescent in-situ hybridization and structural analysis of $5 \mathrm{~S}$ rRNA genes and intergenic spacer sequences in sugar beet (Beta vulgaris). Theor. Appl. Genet. 88, 629-636.

Seibt, K.M., Schmidt, T., and Heitkam, T. (2018). FlexiDot: Highly customizable, ambiguity-aware dotplots for visual sequence analyses. Bioinformatics 34, 3575-3577. 
Stevens, K.A., Wegrzyn, J., Zimin, A., Puiu, D., Crepeau, M., Cardeno, C., Paul, R., Gonzalez-Ibeaz, D., Koriabine, M., Holtz-Morris, A., Martinez-Garcia, P., Sezen, U., Marcais, G., Jermstad, K., Mcguire, P., Loopstra, C.A., Davis, J.M., Eckert, A., Dejong, P., Yorke, J.A., Salzberg, S.L., Neale, D.B., and Langley, C.H. (2016). Sequence of the sugar pine megagenome. Genetics.

Sultana, N., Menzel, G., Heitkam, T., Schmidt, T., Kojima, K.K., Bao, W., and Serçe, S. (2020). Bioinformatics and molecular analysis of satellite repeat diversity in Vaccinium genomes. Genes 11, 527.

Utsunomia, R., Ruiz-Ruano, F.J., Silva, D.M.Z.A., Serrano, É.A., Rosa, I.F., Scudeler, P.E.S., Hashimoto, D.T., Oliveira, C., Camacho, J.P.M., and Foresti, F. (2017). A glimpse into the satellite DNA library in Characidae fish (Teleostei, Characiformes). Front. Genet. 8, 103.

Vondrak, T., Ávila Robledillo, L., Novák, P., Koblížková, A., Neumann, P., and Macas, J. (2020). Characterization of repeat arrays in ultra-long nanopore reads reveals frequent origin of satellite DNA from retrotransposon-derived tandem repeats. Plant J. 101, 484-500.

Wegrzyn, J.L., Liechty, J.D., Stevens, K.A., Wu, L.-S., Loopstra, C.A., Vasquez-Gross, H.A., Dougherty, W.M., Lin, B.Y., Zieve, J.J., Martínez-García, P.J., Holt, C., Yandell, M., Zimin, A.V., Yorke, J.A., Crepeau, M.W., Puiu, D., Salzberg, S.L., De Jong, P.J., Mockaitis, K., Main, D., Langley, C.H., and Neale, D.B. (2014). Unique features of the loblolly pine (Pinus taeda L.) megagenome revealed through sequence annotation. Genetics 196, 891-909.

Wegrzyn, J.L., Lin, B.Y., Zieve, J.J., Dougherty, W.M., Martínez-García, P.J., Koriabine, M., Holtz-Morris, A., Dejong, P., Crepeau, M., Langley, C.H., Puiu, D., Salzberg, S.L., Neale, D.B., and Stevens, K.A. (2013). Insights into the loblolly pine genome: characterization of BAC and fosmid sequences. PLoS ONE 8, e72439.

Wei, X.X., and Wang, X.Q. (2003). Phylogenetic split of Larix: Evidence from paternally inherited cpDNA trnT-trnF region. Plant Syst. Evol. 239, 67-77.

Weiss-Schneeweiss, H., Leitch, A.R., Mccann, J., Jang, T.-S., and Macas, J. (2015). "Employing next generation sequencing to explore the repeat landscape of the plant genome," in Next-Generation Sequencing in Plant Systematics, eds. E. Hörandl \& M.S. Appelhans.).

Wickham, H. (2016). ggplot2: Elegant graphics for data analysis. New York: SpringerVerlag.

Willard, H.F., and Waye, J.S. (1987). Hierarchical order in chromosome-specific human alpha satellite DNA. Trends Genet. 3, 192-198.

Zhang, S.G., Yang, W.H., Han, S.Y., Han, B.T., Li, M.X., and Qi, L.W. (2010). Cytogenetic analysis of reciprocal hybrids and their parents between Larix leptolepis and Larix gmelinii: Implications for identifying hybrids. Tree Genet. Genomes 6, 405-412.

Zhang, T., Talbert, P.B., Zhang, W., Wu, Y., Yang, Z., Henikoff, J.G., Henikoff, S., and Jiang, J. (2013). The CentO satellite confers translational and rotational phasing on CenH3 nucleosomes in rice centromeres. Proc. Natl. Acad. Sci. USA 110, E4875-E4883.

Zonneveld, B.J.M. (2012). Conifer genome sizes of 172 species, covering 64 of 67 genera, range from 8 to 72 picogram. Nord. J. Bot. 30, 490-502.

Zuccolo, A., Scofield, D.G., De Paoli, E., and Morgante, M. (2015). The Ty1-copia LTR retroelement family PARTC is highly conserved in conifers over 200MY of evolution. Gene 568, 89-99. 
bioRxiv preprint doi: https://doi.org/10.1101/2021.03.21.436054; this version posted March 22. 2021. The copyriaht holder for this preprint (which was not certified by peer review) is the author/funder, who has granted bioRxiv a license to display the preprint in perpetuity. It is made available under aCC-BY 4.0 International license.

992

Page 30 of 30 
bioRxiv preprint doi: https://doi.org/10.1101/2021.03.21.436054; this version posted March $22,2021$. The copyright holder for this preprint (which was not certified by peer review) is the author/funder, who has granted bioRxiv a license to display the preprint in perpetuity. It is made available under aCC-BY 4.0 International license.
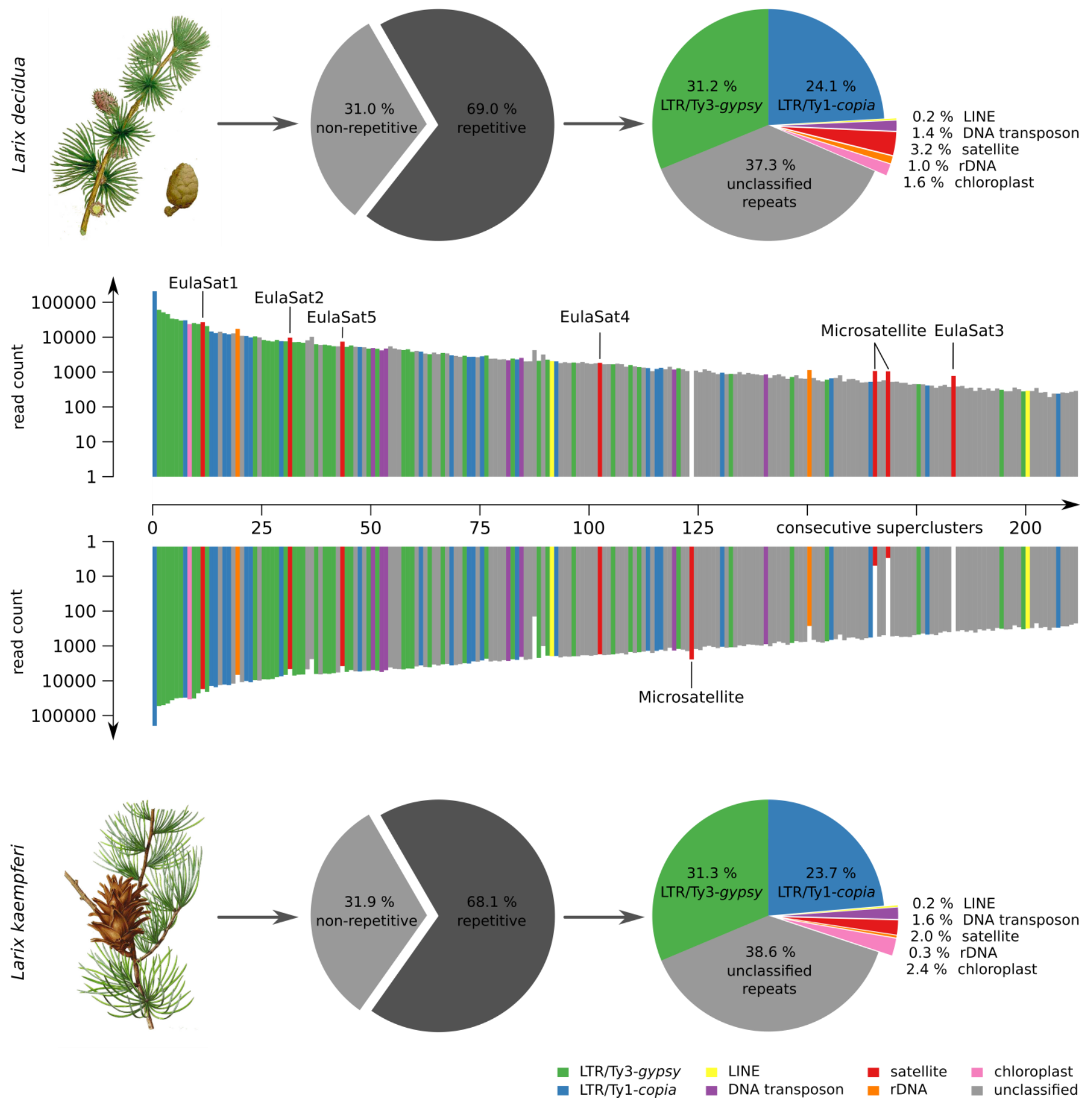

Figure 1: Comparison of repetitive genome fractions reveals high genomic similarity over all repeat types between $L$. decidua and $L$. kaempferi. At the center of each figure, a two-sided barplot shows 214 repeat superclusters with respective read counts in L. decidua (top) and L. kaempferi (bottom). The read count is presented on a logarithmic scale. Experimentally analyzed clusters are marked with the corresponding repeat family name. The composition of each Larix repeat fraction is summarized by pie charts. The plant illustrations are reproduced from Woodville, W., Hooker, W.J., Spratt, G., Medical Botany, 3th edition, vol. 1: (1832) (L. decidua) and M. E.-A. Carriere (ed.) Revue Horticole, serié 4, vol. 40: (1868), Paris (L. kaempferi). 
bioRxiv preprint doi: https://doi.org/10.1101/2021.03.21.436054; this version posted March 22, 2021. The copyright holder for this preprint (which was not certified by peer review) is the author/funder, who has granted bioRxiv a license to display the preprint in perpetuity. It is made available under aCC-BY 4.0 International license.

EulaSat1

EuLaSat2

EulaSat3a

EulaSat3b

EulaSat3c

EulaSat3a

Eulasat3b

EulaSat3c

EulaSat3a Eulasat3b

Eulasat3c

EulaSat3a EulaSat3b

EulaSat3c

EulaSat3a

EulaSat3b

EulaSat3c

EulaSat4a EulaSat4b

Eulasat4a EulaSat4b

Eulasat4a EulaSat4b

EulaSat5

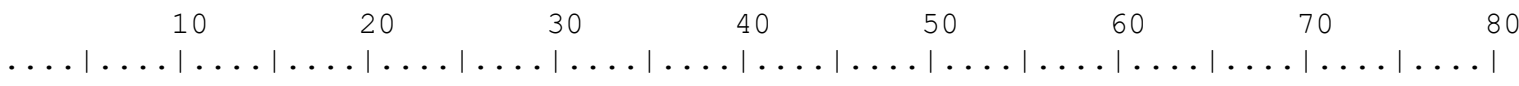

TTTCATAAATGGAATCAACAAAGTATGCACATTCTACGTCATAACGACTTTCTATAGTTTGCGCATGCGTCCGGAAATAA GAAAAGCTTACTTCCCCCGTTTTTTAAAATCACAGCTTCTAGAAGGTTTACATGATTTCTTAAAAAACACGAGTTTTTAG AAAATGTGTTTTA

AAAA AGCT TGAACGTCACGAAAGTTGGCGTGGACGCTTGTCTACCAATGGGACATCCAAATCTATTCTCAAATTTCAA TTCCGAGAAGTTGGTCAAAGTTGAAACTCGACCGTGCGTTTTCGCTAGACTTGGGCTTAAAGGGTGAG 148 bp
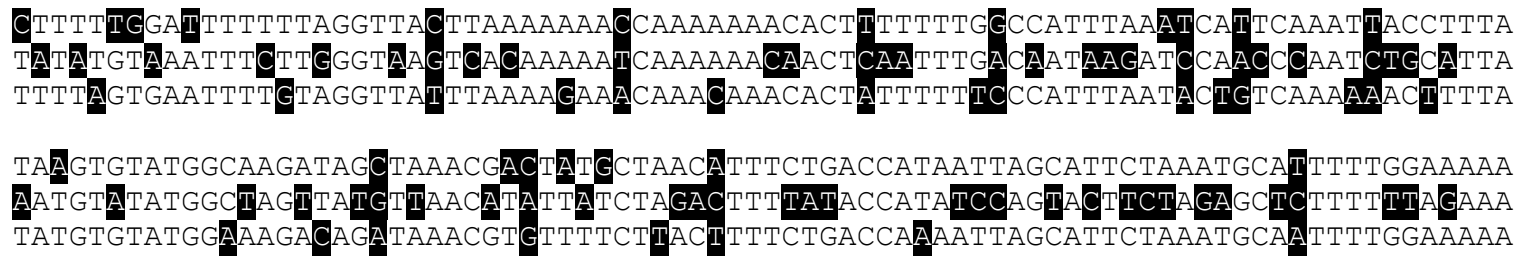

\section{TTCTGAATTTTTAGTGTGATtGTCAGTAGTTGCCACTTCAgtCAAACCATATAAACATTTAAAAATCTAACTTACATT ATAGGAATTTTTAGTGTAATTGTTCAGTAGTTGTCAACACAATTAACCAAGTAAACAACATTTTTTTCTAACTTCTACA ATCTGAATTTTTGTGTTTTGTTCAgTAGTTGCCATCACAGTAAAAACATATAAACATTAAAAAATGTAAGTTATATT}

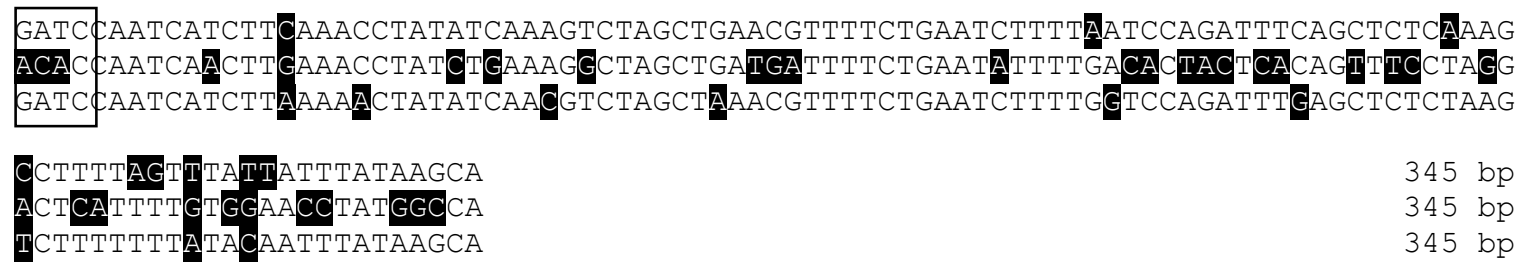

TTCAAAATAGAGCACATGGCACAAGCTCAAGGTATAAGCTAGCAACCACCAATCACCATGGACAGGGTTTTTCTATTGGA TTCAAAATAGAGCACATGGCACAAGCTCAAGGTATAAGCTAGCAACCACCAATCACCATGGACAGGGTTTTTCTATTGGA

AAGCTAGCGACTGCTAGCTTTCCTTCTAGCATAAGCATGTGTGTTTATGCTAGAAGGAAATGCTTATGCTAGAAGGAAAG AAGCTAGCGACTGCTAGCTTTCCTTCTAGCATAAGCATGTGTGTT-ATGCTAGAAGGAAATGCTTATGCTAGAAGGAAAG

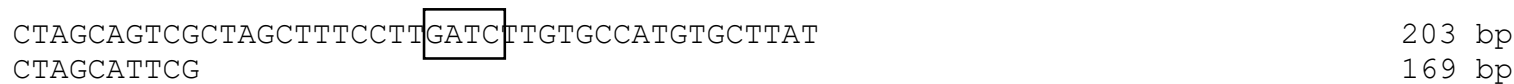

AGTCCAGGGATGATCAAATCCCCTCAACTGTCCACTAAGGACTTCATTCTCGGAGACCTCACGTCTACGGCTCTCTTTAG GACTGAA

Figure 2: Consensus sequences and subunit structure of the tandem repeat monomers. The monomer consensus sequences of the EulaSat1 to EulaSat5 satDNAs are shown. Recognition sites of restriction enzymes used to release the DNA ladder (Figure 3, Figure 5) are indicated by rectangles. HpaI/MspI recognition sites are shaded in grey. EulaSat 3 and EulaSat 4 are divided into the EulaSat3a, EulaSat3b and EulaSat3c as well as the EulaSat4a, and EulaSat $4 \mathrm{~b}$ subfamilies. These sequences are represented as multiple sequence alignment, with ambiguities shaded in black. 
bioRxiv preprint doi: https://doi org/10.1101/2021.03.21.436054; this version posted March 22,2021 . The copyright holder for this preprint (which was not certified by peer review) is the author/funder, who has granted bioRxiv a license to display the preprint in perpetuity. It is made available under aCC-BY 4.0 International license.
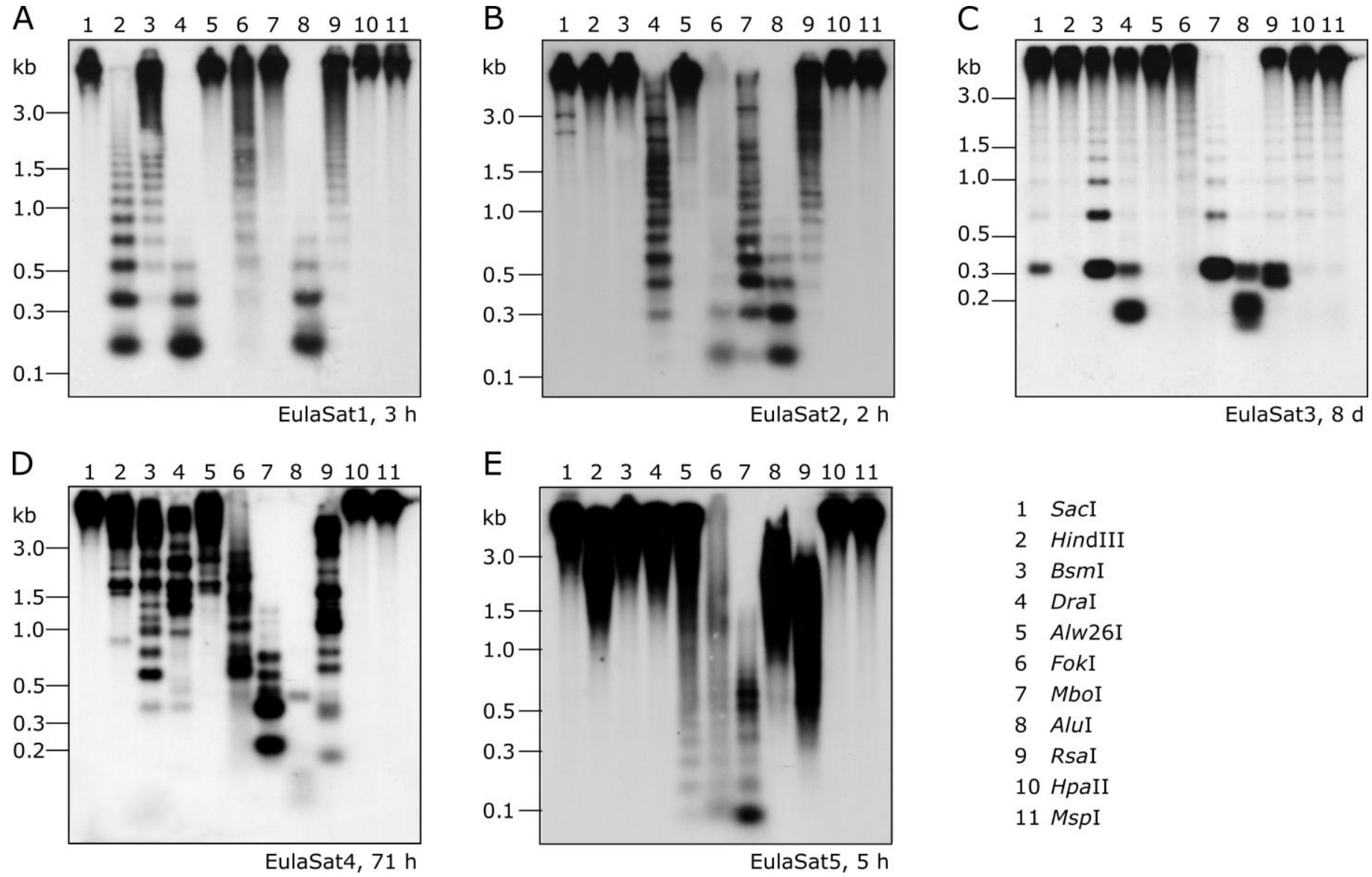

Figure 3: Genomic organization of the Larix satDNA families EulaSat1 to EulaSat5. Southern hybridization of restricted genomic L. decidua DNA with satDNA probes releases the satellite-typical ladder pattern of EulaSat1 (A), EulaSat2 (B), and EulaSat3 (C), EulaSat4 (D), and EulaSat5 (E). The exposition time are indicated in hours (h) or days (d) for each experiment. 
bioRxiv preprint doi: https://doi.org/10.1101/2021.03.21.436054; this version posted March 22, 2021. The copyright holder for this preprint (which was not certified by peer review) is the author/funder, who has granted bioRxiv a license to display the preprint in perpetuity. It is made available under aCC-BY 4.0 International license.

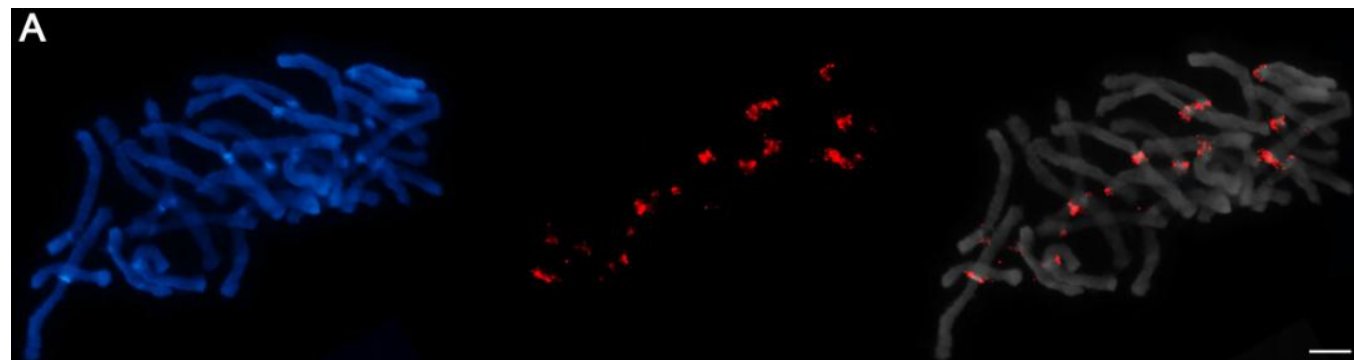

$\mathbf{B}$

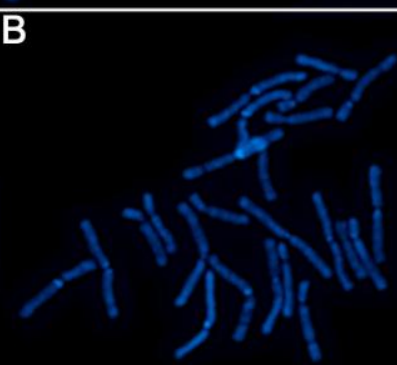

$\mathbf{C}$
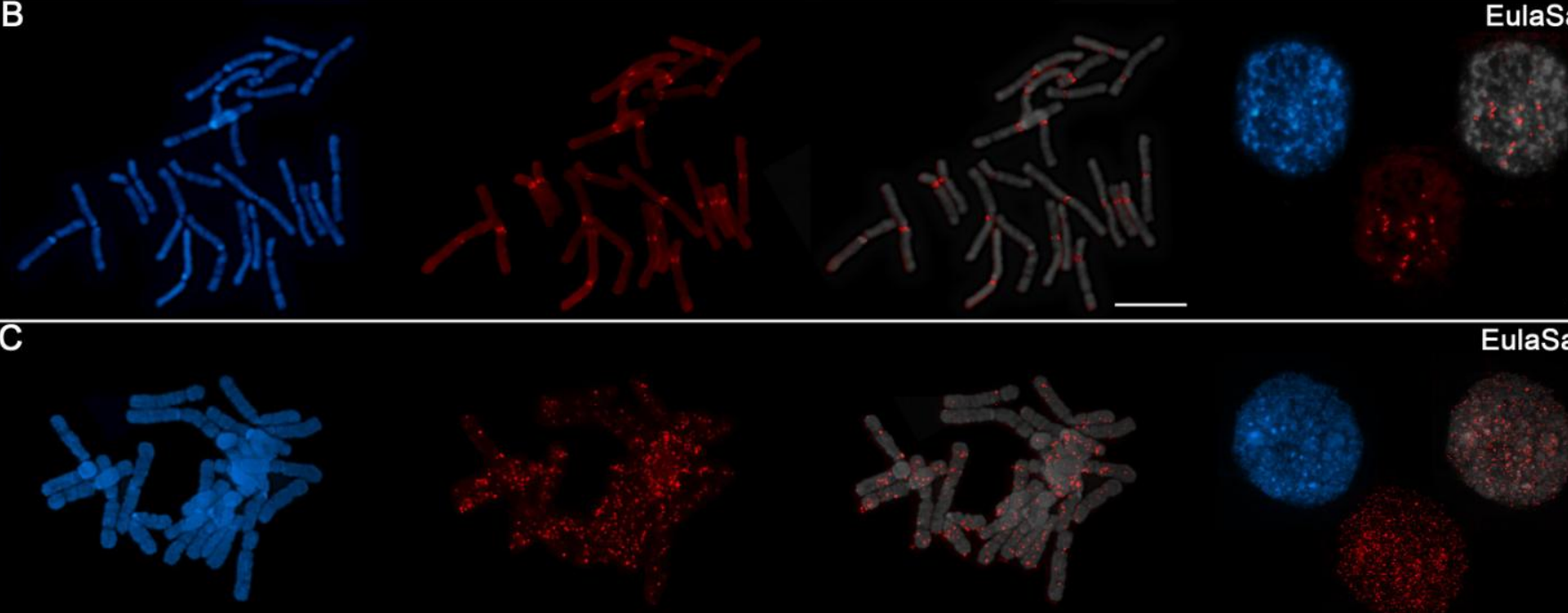

EulaSat3

$\mathbf{D}$
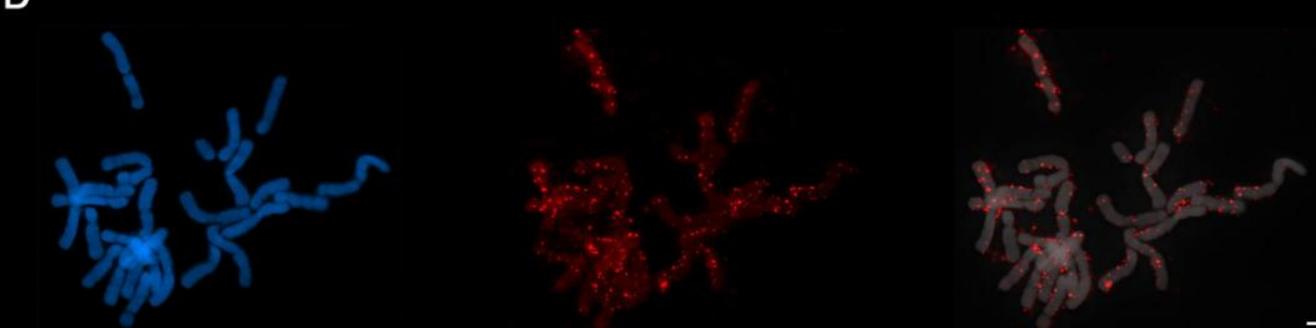

E

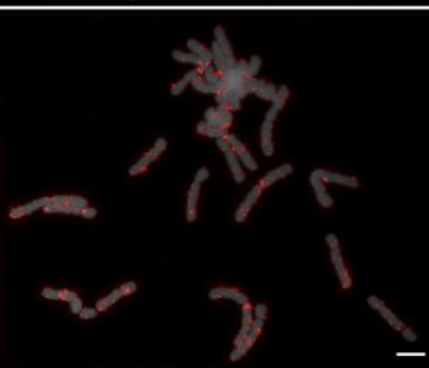

EulaSat5
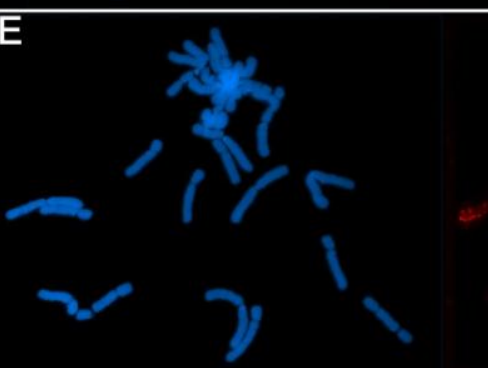

Figure 4: Chromosomal localization of the EulaSat families along Larix decidua chromosomes. Chromosomes have been counterstained with DAPI, indicated in blue and grey. Fluorescent in situ hybridizations of EulaSat1 (A), EulaSat2 (B), EulaSat3 (C), EulaSat4 (D), and EulaSat5 (E) to L. decidua meta- and interphases are shown in red. 
bioRxiv preprint doi: https://doi.org/10.1101/2021.03.21.436054; this version posted March 22,2021 . The copyright holder for this preprint (which was not certified by peer review) is the author/funder, who has granted bioRxiv a license to display the preprint in perpetuity. It is made available under aCC-BY 4.0 International license.

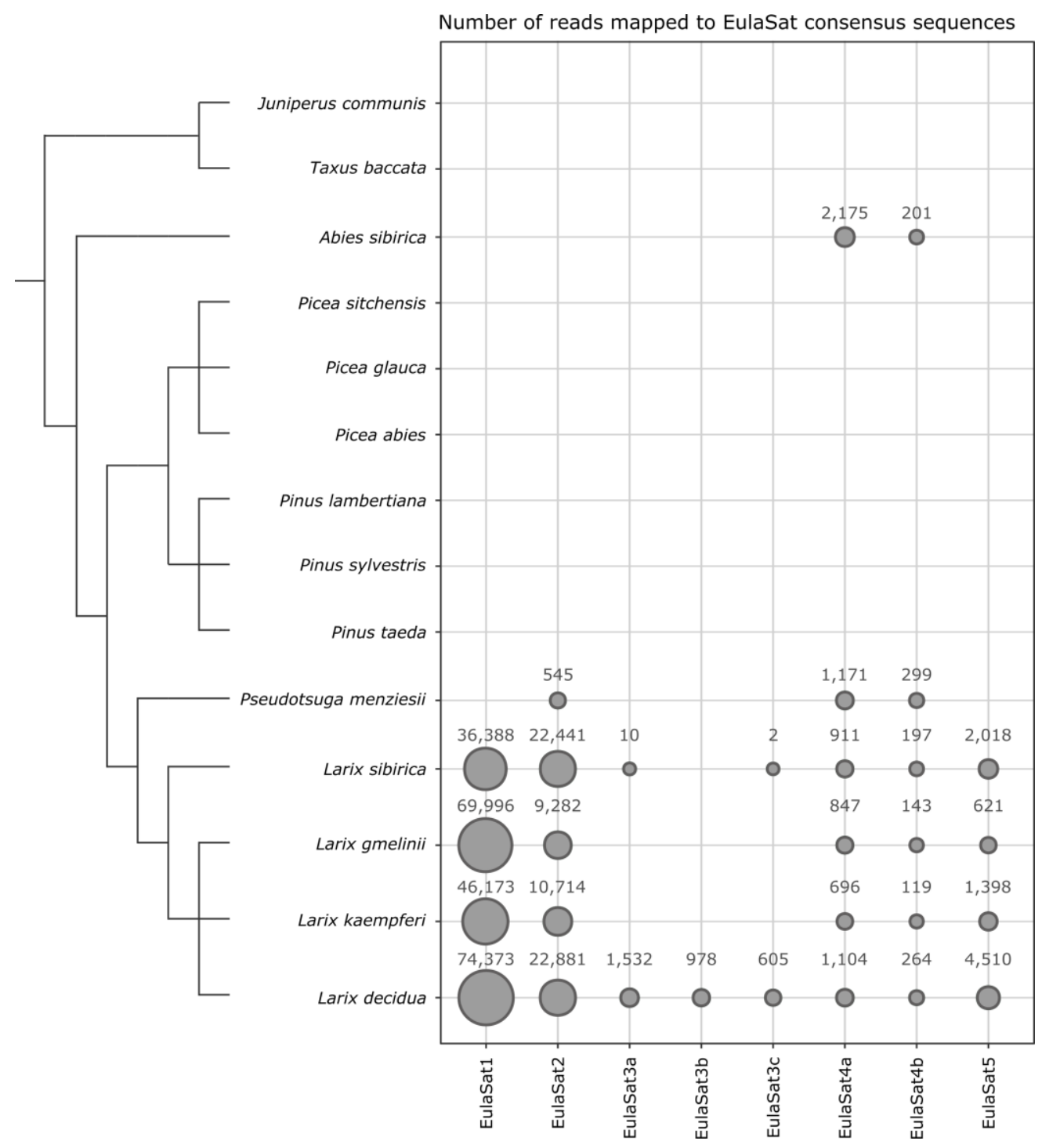

Figure 5: Distribution of the EulaSat tandem repeats in fourteen gymnosperm genomes surveyed by read mapping. The area of each bubble represents the amount of whole genome shotgun Illumina reads mapping to the EulaSat consensus sequences. A total of three million paired reads has been used as input for the mapping analysis. The dendrogram indicates the evolutionary relationship between the species according to Wei et al. (2003) for the genus Larix and Lu et al. (2014) for the overall phylogeny. The branch lengths are not to scale. 

made available under aCC-BY 4.0 International license.
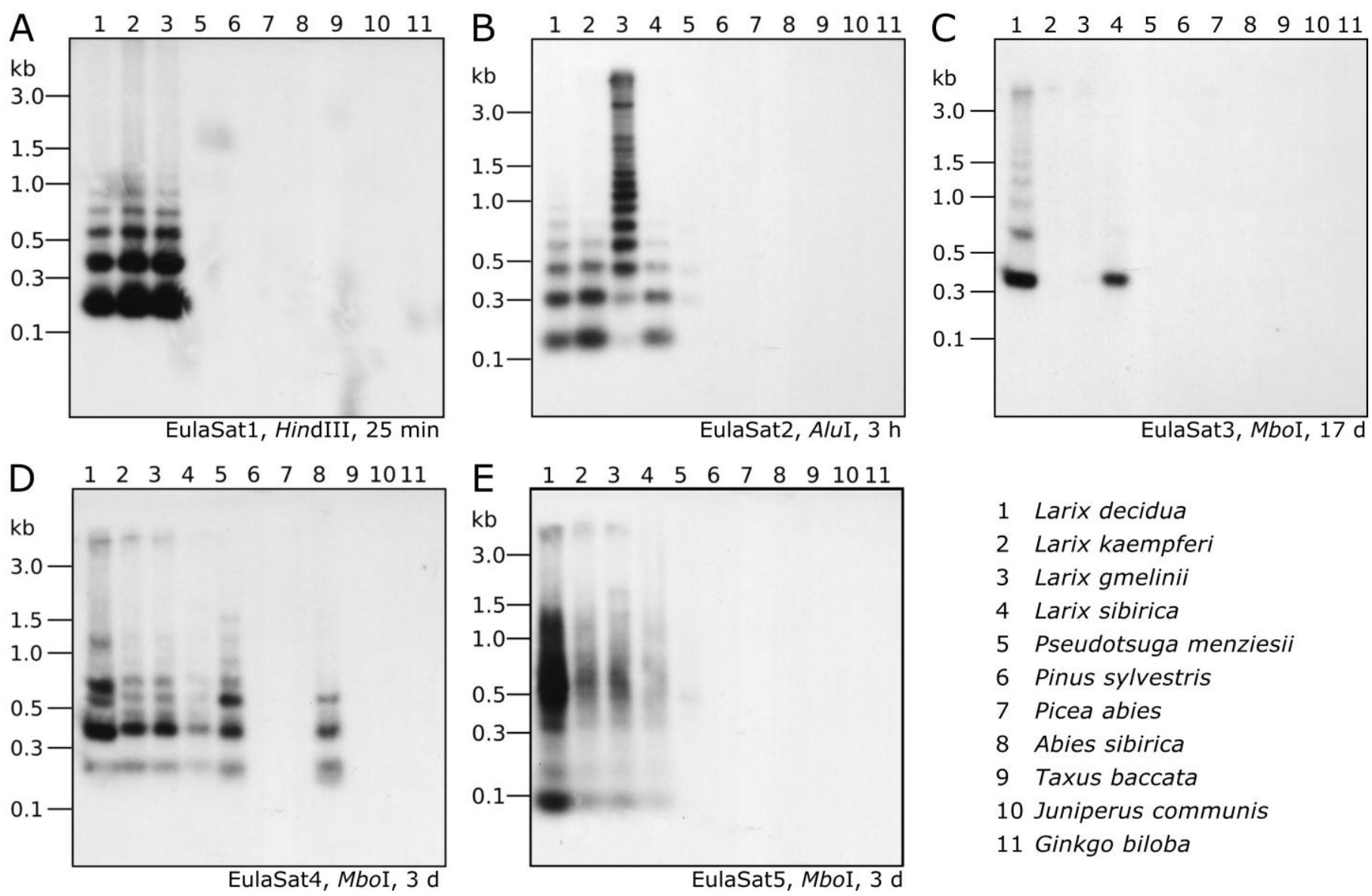

Figure 6: Organization and abundance of EulaSat repeats in related gymnosperm genomes. Genomic DNA of eleven gymnosperms has been restricted as indicated in each panel and was analyzed by comparative Southern hybridization of EulaSat1 (A), EulaSat2 (B), EulaSat3 (C), EulaSat4 (D), and EulaSat5 (E). Exposure times ranged between 25 minutes and seventeen days, as indicated below the autoradiographs. 
bioRxiv preprint doi: https://doi.org/10.1101/2021.03.21.436054; this version posted March 22, 2021. The copyright holder for this preprint (which was not certified by peer review) is the author/funder, who has granted bioRxiv a license to display the preprint in perpetuity. It is made available under aCC-BY 4.0 International license.
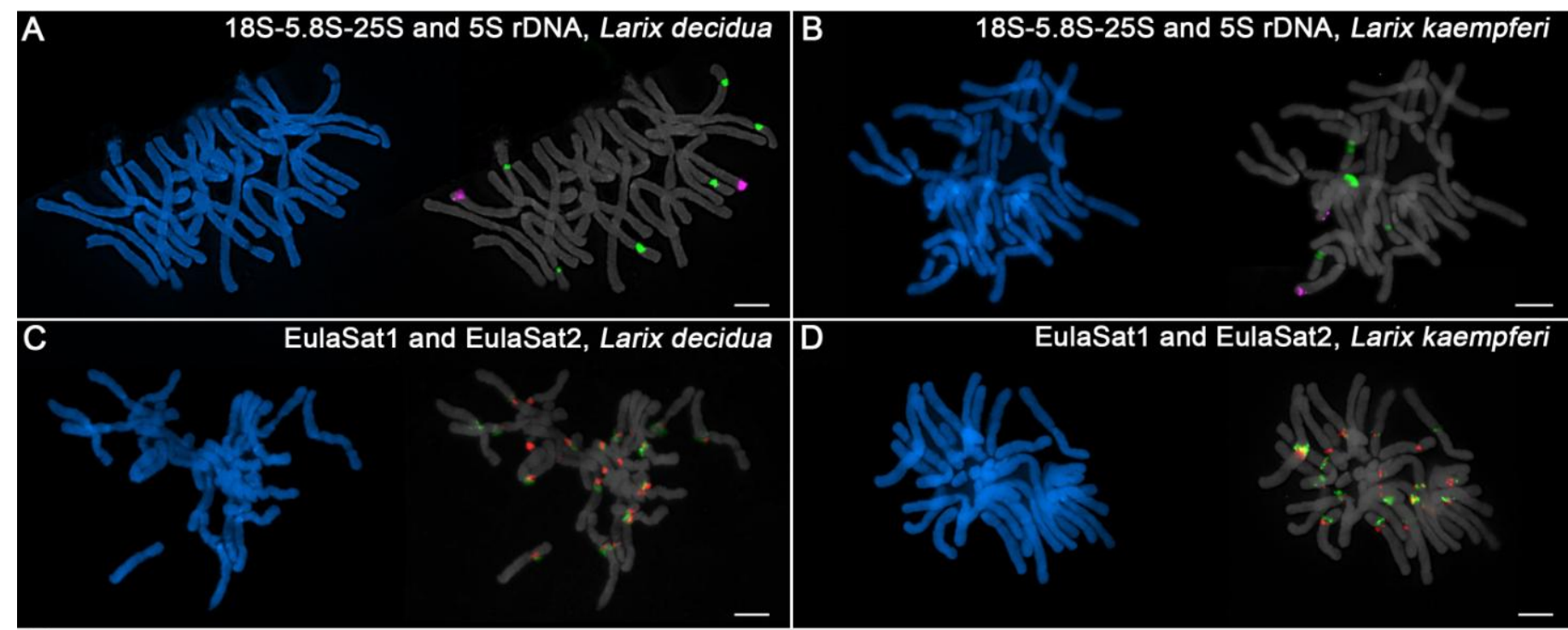

$\bar{E}$

18S-5.8S-25S rDNA, 5S rDNA and EulaSat3, Larix $x$ eurolepis
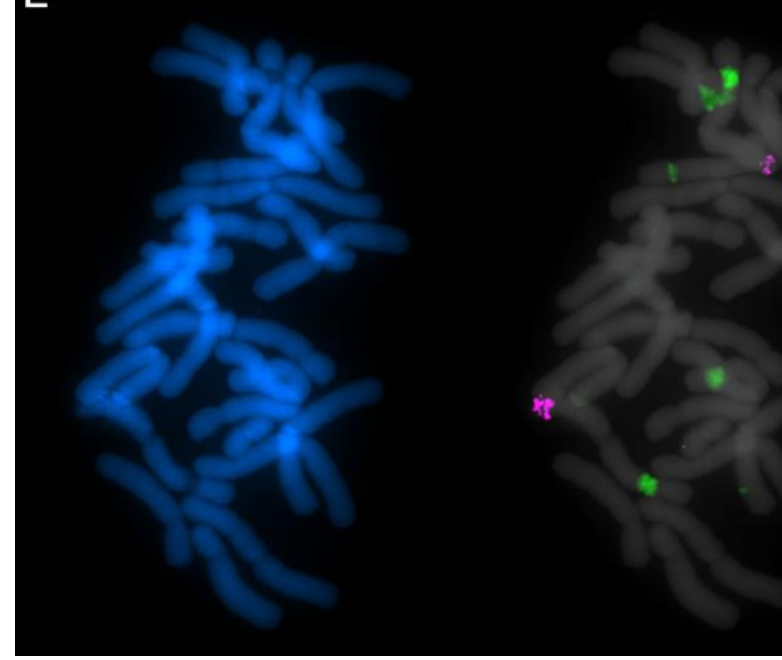

Figure 7: Chromosomal location of rDNAs and the EulaSat families for comparison of L. kaempferi, L. decidua, and $L . \times$ eurolepis. The chromosomes have been counterstained with DAPI, indicated in blue and grey. Reproduced are fluorescent in situ hybridizations of the 5S (magenta) and 18S-5.8S-25S rDNAs (green) to metaphases of $L$. decidua (A) and L. kaempferi (B). EulaSat1 (green) and EulaSat2 (red) were comparatively hybridized along metaphase chromosomes of L. decidua (C) and L. kaempferi (D). The genome-specific EulaSat3 family (red) was hybridized along chromosomes of the interspecific L. $\times$ eurolepis hybrid, along with probes for the $5 \mathrm{~S}$ (magenta) and 18S-5.8S-25S rDNAs (green). 